\title{
ARTICLE
}

\section{A pan-cancer blueprint of the heterogeneous tumor microenvironment revealed by single-cell profiling}

Junbin Qian (iD) ${ }^{1,2}$, Siel Olbrecht ${ }^{1,2,3}$, Bram Boeckx ${ }^{1,2}$, Hanne Vos ${ }^{4}$, Damya Laoui ${ }^{5,6}$, Emre Etlioglu (iD), Els Wauters ${ }^{8,9}$, Valentina Pomella ${ }^{7}$, Sara Verbandt ${ }^{7}$, Pieter Busschaert ${ }^{3}$, Ayse Bassez ${ }^{1,2}$, Amelie Franken $^{1,2}$, Marlies Vanden Bempt ${ }^{1,2}$, Jieyi Xiong ${ }^{1,2}$, Birgit Weynand ${ }^{10}$, Yannick van Herck ${ }^{11}$, Asier Antoranz ${ }^{10}$, Francesca Maria Bosisio ${ }^{10}$, Bernard Thienpont $\mathbb{I D}^{12}$, Giuseppe Floris ${ }^{10}$, Ignace Vergote ${ }^{3}$, Ann Smeets ${ }^{4}$, Sabine Tejpar ${ }^{7}$ and Diether Lambrechts $\mathbb{D}^{1,2}$

The stromal compartment of the tumor microenvironment consists of a heterogeneous set of tissue-resident and tumor-infiltrating cells, which are profoundly moulded by cancer cells. An outstanding question is to what extent this heterogeneity is similar between cancers affecting different organs. Here, we profile 233,591 single cells from patients with lung, colorectal, ovary and breast cancer $(n=36)$ and construct a pan-cancer blueprint of stromal cell heterogeneity using different single-cell RNA and protein-based technologies. We identify 68 stromal cell populations, of which 46 are shared between cancer types and 22 are unique. We also characterise each population phenotypically by highlighting its marker genes, transcription factors, metabolic activities and tissue-specific expression differences. Resident cell types are characterised by substantial tissue specificity, while tumor-infiltrating cell types are largely shared across cancer types. Finally, by applying the blueprint to melanoma tumors treated with checkpoint immunotherapy and identifying a naïve $\mathrm{CD} 4^{+}$T-cell phenotype predictive of response to checkpoint immunotherapy, we illustrate how it can serve as a guide to interpret scRNA-seq data. In conclusion, by providing a comprehensive blueprint through an interactive web server, we generate the first panoramic view on the shared complexity of stromal cells in different cancers.

Cell Research (2020) 30:745-762; https://doi.org/10.1038/s41422-020-0355-0

\section{INTRODUCTION}

In recent years, single-cell RNA sequencing (scRNA-seq) studies have provided an unprecedented view on how stromal cells consist of heterogeneous and phenotypically diverse populations of cells. Indeed, by now, the tumor microenvironment (TME) of several cancer types has been profiled, including melanoma, ${ }^{1}$ lung cancer, ${ }^{2}$ head and neck cancer, ${ }^{3}$ hepatocellular carcinoma, ${ }^{4}$ glioma, ${ }^{5}$ medulloblastoma, ${ }^{6}$ pancreatic cancer, ${ }^{7}$ etc. However, while there is still an unmet need to chart TME heterogeneity in additional tumors and cancer types, the higher-level question relates to the similarities between these microenvironments.

Indeed, it remains unexplored whether the same stromal cell phenotypes are present in different cancer types. Also, it is not clear to what extent these phenotypes are reminiscent of the normal tissue from which they originate and are thus characterised by tissue-specific expression. Such knowledge is highly desirable, because it not only facilitates comparison between different scRNA-seq studies, but also contributes to our insights in cancer type-specific gene expression patterns and treatment vulnerabilities.
Furthermore, this knowledge would allow us to assess at singlecell level the underlying mechanisms of action of novel cancer therapies. Indeed, most innovative cancer therapies are given to cancer patients with advanced disease, in which tissue biopsies often can only be collected from metastasized organs. It is difficult, however, to systematically identify stromal phenotypes in biopsies taken from different organs, as their expression is determined by the metastasized tissue. Another challenge is that rare stromal cell phenotypes often cluster together with other more common phenotypes, and can therefore only be detected when several 10,000 s of cells derived from multiple patient biopsies are profiled together. Many of these rare phenotypes are critical in determining response to cancer treatment and therefore need to be assessed as a separate population of cells. For instance, scRNA-seq of melanoma T-cells exposed to anti-PD1 identified $\mathrm{TCF}^{+} \mathrm{CD}^{+}$ memory-precursor T-cells as the population underlying treatment response. These cells are rare, as they represent only $\sim 15 \%$ of $\mathrm{CD}^{+} \mathrm{T}$-cells, which by themselves represent only $\sim 2.5 \%$ of cells in these tumors. ${ }^{8}$ In order not to miss these rare phenotypes, a

\footnotetext{
${ }^{1}$ VIB Center for Cancer Biology, Leuven, Belgium; ${ }^{2}$ Laboratory for Translational Genetics, Department of Human Genetics, KU Leuven, Leuven, Belgium; ${ }^{3}$ Department of Obstetrics and Gynaecology, University Hospitals Leuven, Leuven, Belgium; ${ }^{4}$ Department of Oncology, KU Leuven, Surgical Oncology, University Hospitals Leuven, Leuven, Belgium; ${ }^{5}$ Lab of Cellular and Molecular Immunology, Vrije Universiteit Brussel, Brussels, Belgium; ${ }^{6}$ Myeloid Cell Immunology Lab, VIB Center for Inflammation Research, Brussels, Belgium; ${ }^{7}$ Laboratory of Molecular Digestive Oncology, Department of Oncology, KU Leuven, Leuven, Belgium; ${ }^{8}$ Respiratory Oncology Unit (Pneumology) and Leuven Lung Cancer Group, University Hospital KU Leuven, Leuven, Belgium; ${ }^{9}$ Laboratory of Pneumology, Department of Chronic Diseases, Metabolism and Ageing, KU Leuven, Leuven, Belgium; ${ }^{10}$ Department of Imaging and Pathology, Laboratory of Translational Cell \& Tissue Research and University Hospitals Leuven, Department of Pathology, KU Leuven-University of

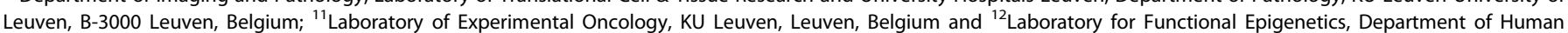
Genetics, KU Leuven, Leuven, Belgium

Correspondence: Diether Lambrechts (Diether.Lambrechts@kuleuven.vib.be)
}

Received: 9 October 2019 Accepted: 5 May 2020

Published online: 19 June 2020 
blueprint of the different cell populations present in each cancer type would be of considerable benefit.

We therefore generated a comprehensive blueprint of stromal cell heterogeneity across cancer types and provide a detailed view on the shared complexity and heterogeneity of stromal cells in these cancers. We illustrate how this blueprint can serve as a guide to interpret scRNA-seq data at individual patient level, even when comparing tumors collected from different tissues or profiled using different scRNA-seq technologies. Our single-cell blueprint can be visualised, analysed and downloaded from an interactive web server (http://blueprint.lambrechtslab.org).

\section{RESULTS}

scRNA-seq and cell typing of tumor and normal tissue

First, we performed scRNA-seq on tumors from 3 different organs (or cancer types): colorectal cancer (CRC, $n=7)$ ) lung cancer (LC, $n=8)$ and ovarian cancer ( $\mathrm{OvC}, n=5)$. Whenever possible, we retrieved both malignant (tumor) and matched non-malignant (normal) tissue during surgical resection with curative intent. All tumors were treatment-naïve and reflected different disease stages (e.g., stage I-IV CRC) or histopathologies (e.g., adenocarcinoma versus squamous LC), and whenever possible tissues were collected from different anatomic sites (e.g., primary tumor from the ovary and omentum in $\mathrm{OvC}$, or from core versus border regions in (RC). Overall, 50 tumor tissues and 17 normal tissues were profiled (Fig. 1a). Clinical and tumor mutation data are summarised in Supplementary information, Tables S1-3.

Following resection, tissues were rapidly digested to a singlecell suspension and unbiasedly subjected to $3^{\prime}$-scRNA-seq. After quality filtering (Materials and methods), we obtained $\sim 1$ billion unique transcripts from 183,373 cells with > 200 genes detected. Of these, $71.7 \%$ of cells originated from malignant tissue. Principle component analysis (PCA) using variably expressed genes was used to generate t-SNEs at different resolutions (Supplementary information, Fig. S1a, b). Marker genes were used to identify cell types (Supplementary information, Fig. S1c). At low resolution, cells clustered based on cancer type, whereas at high resolution they clustered based on patient identity (Supplementary information, Fig. S1d). Also, when assessing how cell types previously identified in LC now clustered, ${ }^{2}$ obvious differences were noted, with similar phenotypic cells now belonging to distinct clusters.

\section{Sub-phenotyping of cell types}

We therefore used a different strategy. First, we clustered cells for each cancer type separately and assigned cell type identities to each cell (Fig. 1a). The result revealed that cells mostly clustered based on cell type (Fig. 1b; Supplementary information, Fig. S1e), allowing us to assess the relative contribution of tumor versus normal tissue or individual patients to each cell type (Fig. 1c-e; Supplementary information, Fig. S1f). We observed that dendritic cells were transcriptionally most active, while T-cells were the most frequent cell type across cancer types (Fig. 1e, f), especially in LC (as observed in other datasets; Supplementary information, Fig. S1g). We also identified cell types specific for one cancer type, including lung alveolar, epithelial and enteric glial cells.

Next, we pooled cells from different cancer types based on cell type identity and performed PCA-based unaligned clustering, generating t-SNEs displaying the phenotypic heterogeneity for each cell type (Fig. 1a). For alveolar, epithelial and enteric glial cells this generated 15 tissue-specific subclusters (LC: 5 alveolar clusters and 1 epithelial cluster; CRC: 8 epithelial clusters and 1 enteric glial cluster), most of which have been described previously 9,10 (Supplementary information, Fig. S1h-p). Additionally, 7 tissuespecific subclusters were identified amongst the fibroblasts and macrophages (see below). Separately, we performed canonical correlation analysis (CCA) for each cancer type followed by graphbased clustering to generate a t-SNE per cell type ${ }^{11}$ (Fig. 1a). To avoid that CCA would erroneously assign cells unique for a cancer type, we did not include any of the 22 tissue-specific subclusters. Thus, while unaligned clustering revealed patient or cancer typespecific clusters, CCA aligned common sources of variation between cancer types. Two measures to calculate sample bias (i.e., "Shannon index" and "mixing metrics", see "Materials and methods") confirmed that after CCA bias decreased in all clusters (Supplementary information, Fig. S1q, r).

Overall, we identified 68 stromal subclusters or phenotypes, of which 46 were shared across cancer types. The number of phenotypes varied between cell types, ranging between 5 to 11 for dendritic cells and fibroblasts, respectively. Our approach was less successful for cancer cells, which due to underlying genetic heterogeneity continued to cluster patient-specifically (Supplementary information, Fig. S1s-u). The number of cancer cells varied substantially between tumors, while also T-cells, myeloid cells and B-cells varied considerably (Supplementary information, Fig. S1v-w).

Below, we describe each stromal phenotype in more detail, highlighting the number of cells, read counts and transcripts across all cancer types and for each cancer type separately, both in tumor versus normal tissue (Supplementary information, Table S4). Additionally, marker genes and functional characteristics of each phenotype are highlighted (Supplementary information, Table S5). The enrichment or depletion of these phenotypes in a cancer type (LC, CRC and OvC) or tissue (tumor versus normal) are evaluated (Supplementary information, Table S6), while gene set enrichment analysis for biological and disease pathways (REACTOME and Gene Ontology) is also performed (see http://blueprint. lambrechtslab.org).

Endothelial cells, tissue-specificity confined to normal tissue Clustering the transcriptomes of 8223 endothelial cells (ECs) using unaligned and CCA-aligned approaches identified, respectively, 13 and 9 clusters, each with corresponding marker genes (Fig. 2a-C; Supplementary information, Fig. S2a-c). Five CCA-aligned clusters were shared between cancer types (Fig. 2d, e), including, based on marker gene expression, C1_ESM1 tip cells (ESM1, NID2), C2_ACKR1 high endothelial venules (HEVs) and venous ECs (ACKR1, SELP), C3_CA4 capillary (CA4, CD36), C4_FBLN5 arterial (FBLN5, GJA5) and C5_PROX1 lymphatic (PROX1, PDPN) ECs. Three other clusters displayed T-cell (C6_CD3D), pericyte (C7_RGS5) and myeloid-specific (C8_AIF1) marker genes and consisted of doublet cells, while one cluster consisted of low-quality ECs (C9; Supplementary information, Fig. S2d, e). Tip ECs only resided in malignant tissue and were most prevalent in CRC, while also HEVs were enriched in tumors. In contrast, capillary ECs (cECs) were enriched in normal tissue (Fig. 2d-f; Supplementary information, Fig. S2f). We identified several genes differentially expressed between tumor and normal tissue (Supplementary information, Fig. S2g and Table S7). For instance, the pro-angiogenic factor perlecan (or HSPG2) was highly expressed in tumor versus normal cECs.

There were 5 unaligned $\mathrm{CEC}$ clusters, which clustered together (in C3_CA4) after CCA. Among these, 4 were derived from normal tissue (NEC1-4; Fig. 2g). Moreover, NEC1-3s were all from lung, suggesting that most $\mathrm{CEC}$ heterogeneity is ascribable to normal lung. C3_NEC1s represented alveolar CECs based on the absence of VWF, while C3_NEC2s and C3_NEC3s represented extra-alveolar $\mathrm{CECs}^{12,13}$ (Fig. 2g-i). C3_NEC1s expressed EDNRB, an oxygensensitive regulator mediating vasodilation, ${ }^{14}$ but also IL33receptor IL1RL1 (ST2). This is surprising as major IL-33 effector cell types are thus far only immune cells, including basophils and innate lymphocytes. ${ }^{10}$ Both extra-alveolar cNEC clusters expressed EDN1, which is a potent vasoconstrictor. C3_NEC3s additionally expressed cytokines, chemotactic and immune cell homing molecules (e.g., IL6, CCL2, ICAM1) (Supplementary information, Fig. S2h). In contrast, C3_NEC4s were exclusively composed of 
a

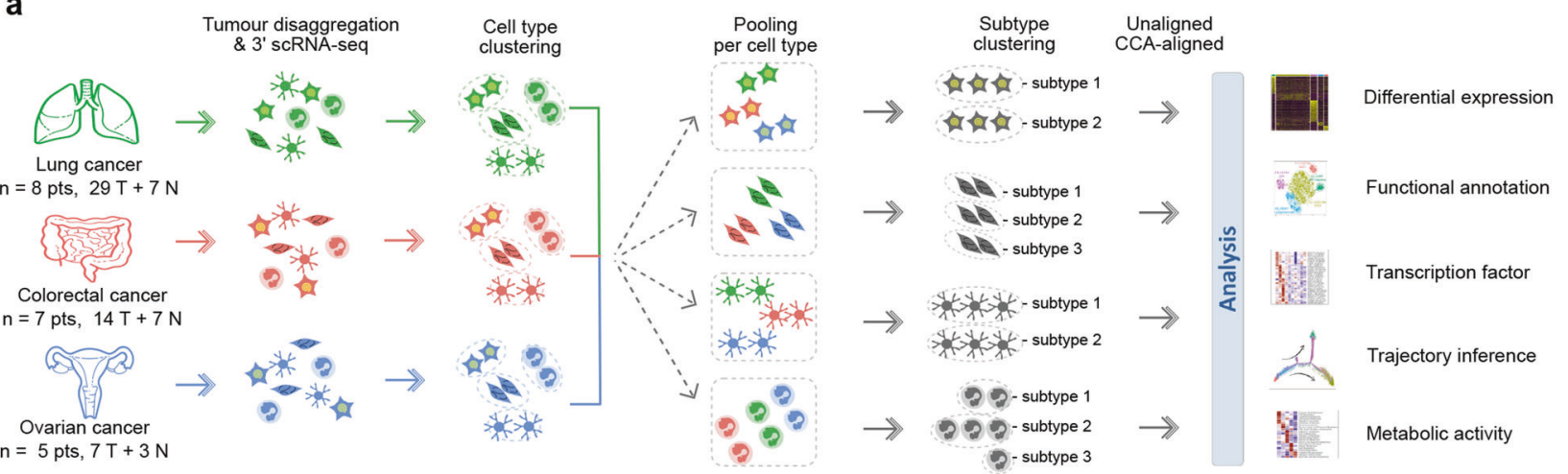

b
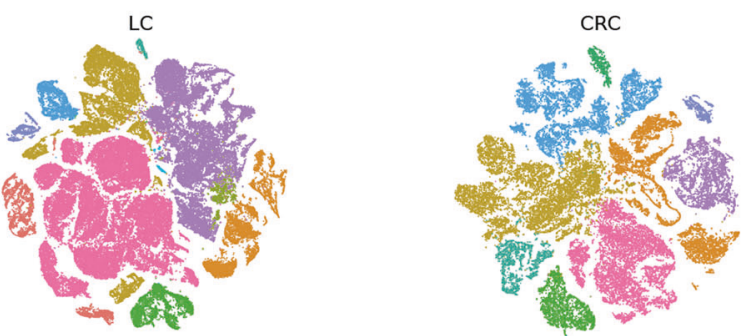

C
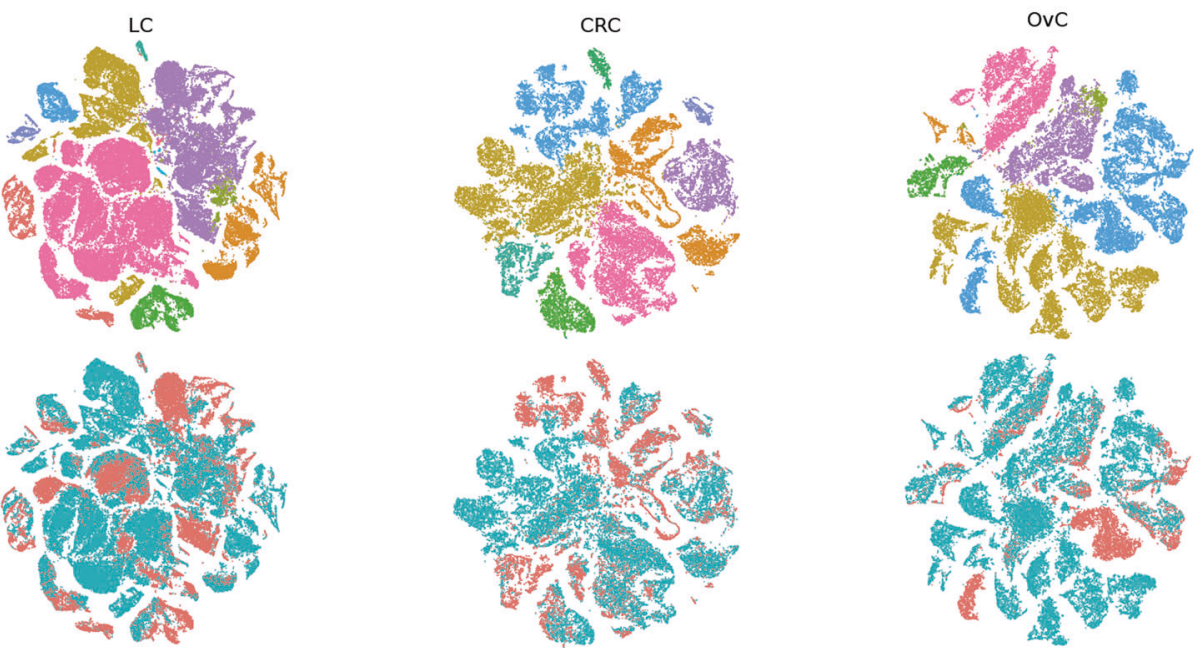

Cell type

Alveolar Epithelial cell

B-cell Erythroblast

Cancer cell Fibroblast

Dentritic cell Mast cell

Endothelial cell Myeloid cell

Entric glia T-cell
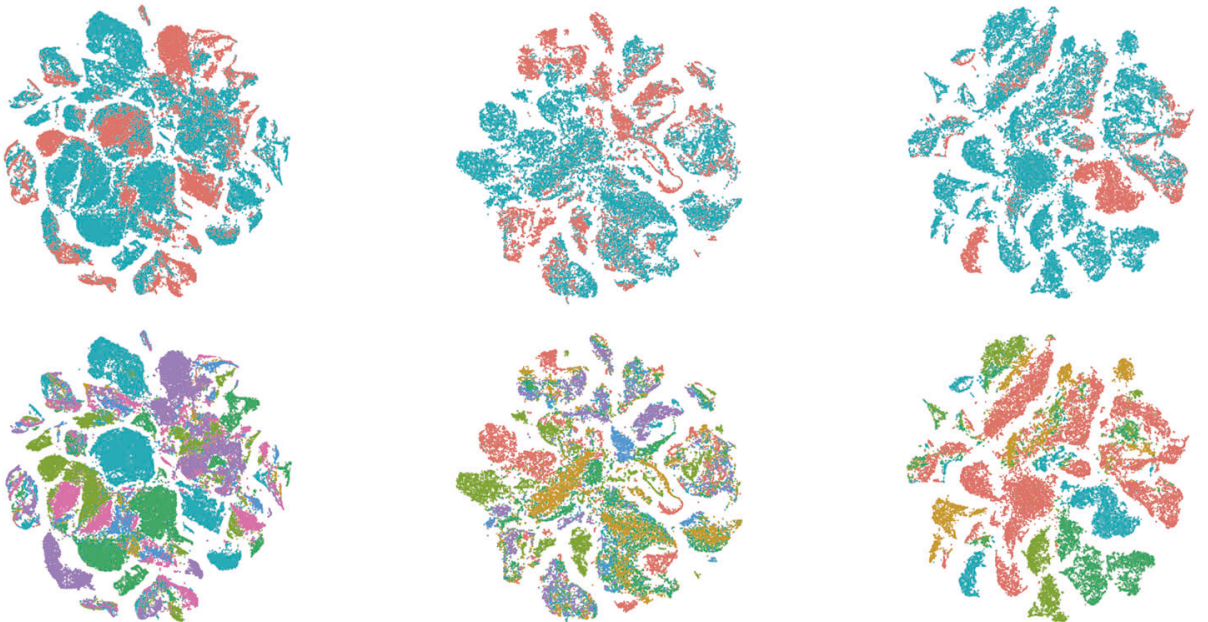

Sample origin

- Normal

Tumour
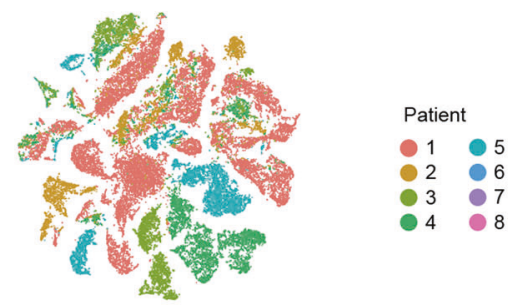

e
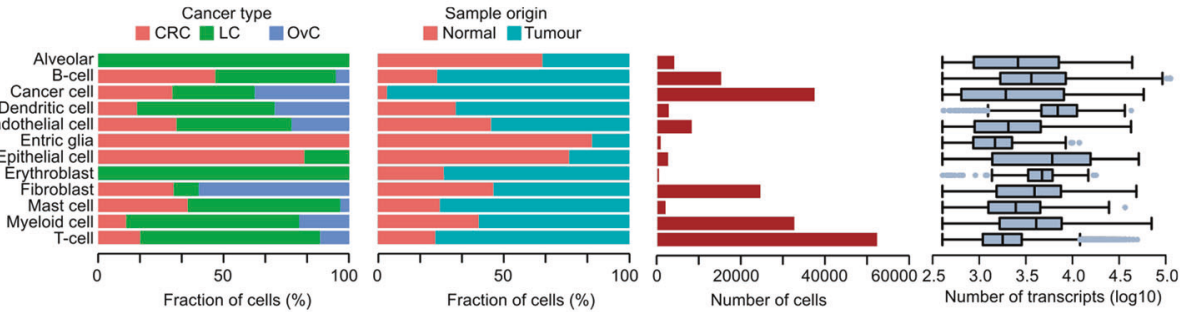

f

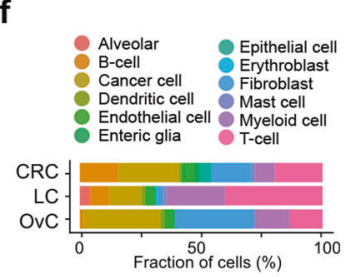

Fig. 1 Experimental design and cell typing. a Analysis workflow of tumor and matched normal samples from 3 cancer types. b-d t-SNE representation for LC $(n=93,576$ cells), CRC $(n=44,685)$ and OvC $(45,115)$. Colour-coded for cell type (b), sample origin (c) and patient (d). e Bar plots representing per cell type from left to right: the fraction of cells per tissue and per origin, the number of cells, the total number of transcripts. Dendritic cells were transcriptionally most active $\left(P<1.6 \times 10^{-10}\right)$. f Fraction of cells for major cell types per cancer type. T-cells were most frequent in LC $(P<0.0047)$.

ovary and colon-derived cells, suggesting similarities between NECs from both tissues. A polarized distribution of ovary and colon-derived ECs within the C3_NEC4 cluster (Fig. 2g) suggests, however, that there are also differences between both tissues. In contrast, tumor cECs (C3_TECs) were derived from all 3 cancer types and lacked tissue specificity on the t-SNE. Indeed, C3_TECs were all characterised by tumor EC markers PLVAP and IGFBP $7^{15-17}$
(Supplementary information, Fig. S2h and Table S5), and only few genes were differentially expressed between cancer types in TECs (Supplementary information, Fig. S2i).

SCENIC ${ }^{18}$ identified different transcription factors (TFs) underlying each EC phenotype (Fig. 2j, k; Supplementary information, Table S8). For instance, activation of NF-KB (NFKB1) and HOXB pathways was confined to C3_NEC3s and C3_TECs, respectively. 
a

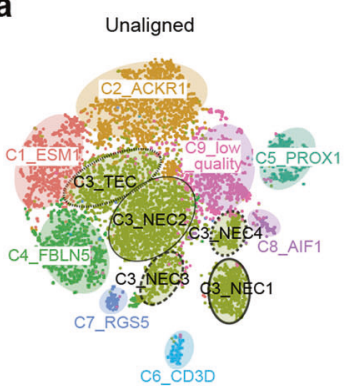

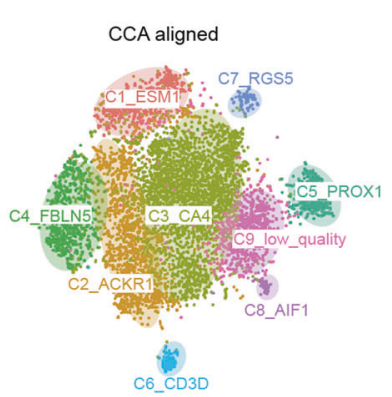

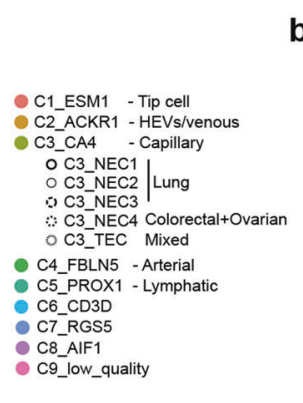

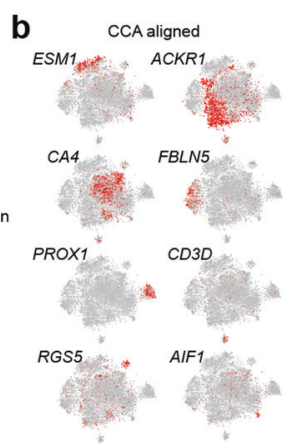

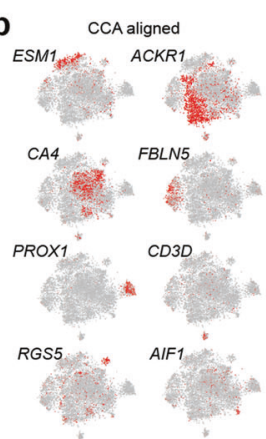
C ESM1 $\frac{1}{1}\lfloor\perp, \perp \perp \perp \perp$ NID2 $\downarrow_{1} \perp_{\perp+\downarrow} \downarrow$ ACKR1 $\perp \Omega \perp \perp \perp \perp \perp \perp$ CA4 $\perp \ell \mid \downarrow \ell \perp L$

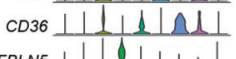
FBLN5 $\perp \perp\lfloor\perp \perp \perp \perp$ GJA5 $\frac{\perp \perp \Omega_{1} \perp \perp \perp}{\text { PROX } 1+1}$

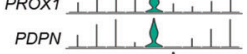
CD3D $\frac{\perp \perp \perp \Omega_{1} \perp}{\perp}$

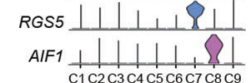
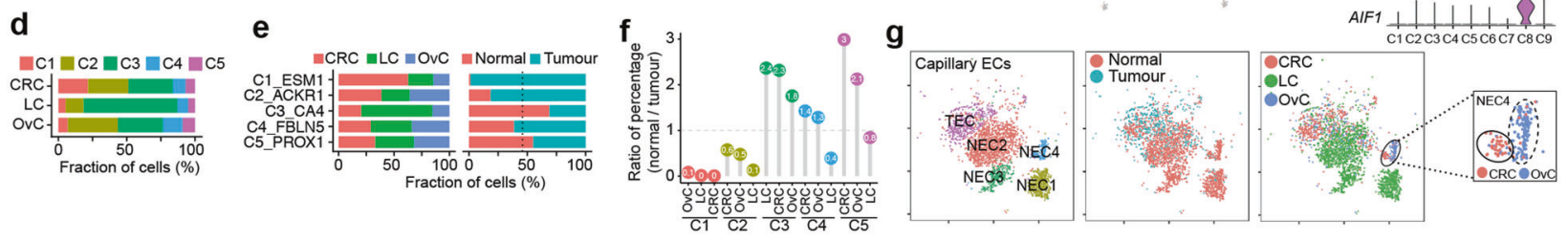

h
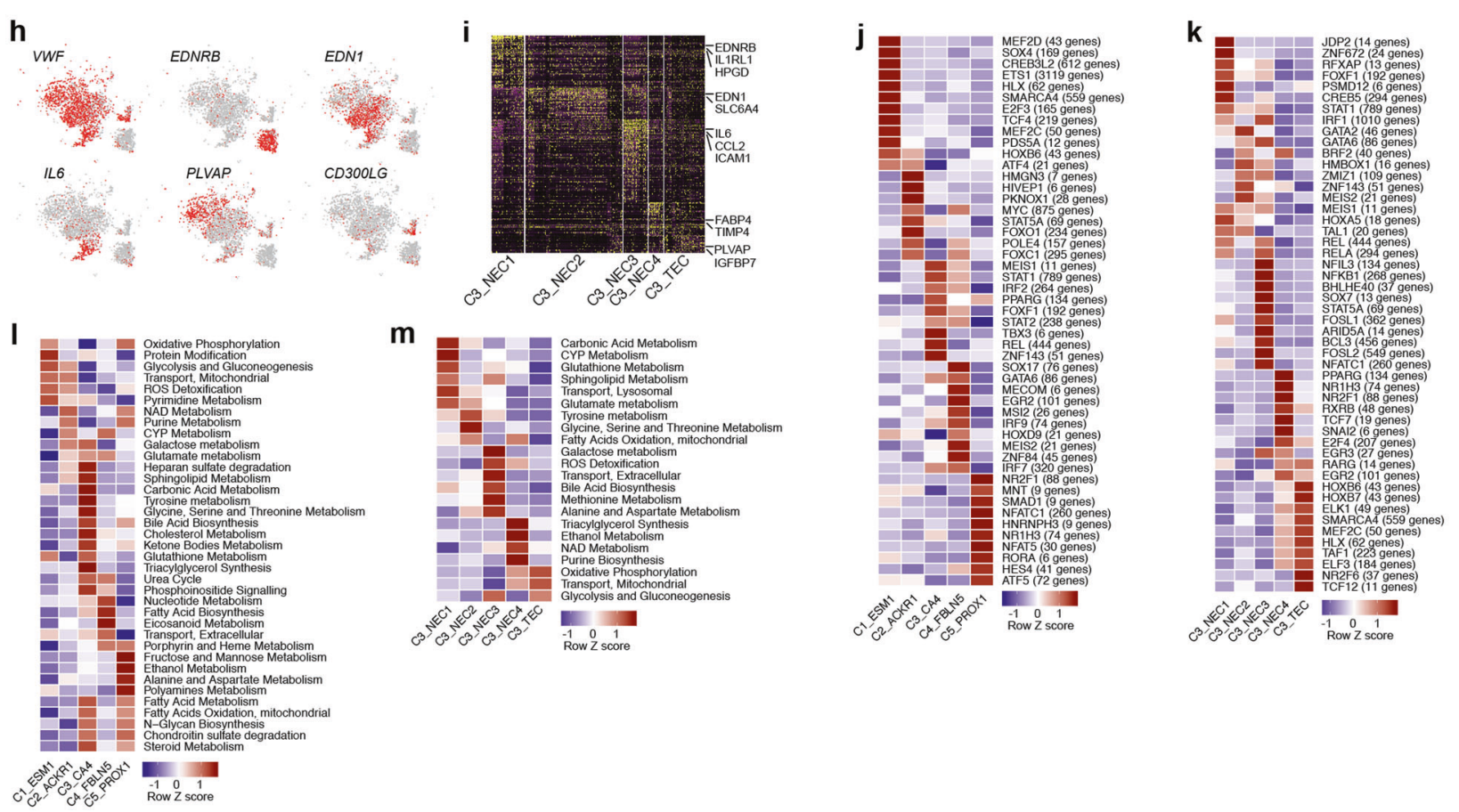

Fig. 2 Clustering 8223 ECs. a t-SNEs colour-coded for annotated ECs by unaligned and CCA-aligned clustering. $\mathbf{b}$ t-SNEs with EC marker gene expression for CCA clusters. c Marker gene expression per EC cluster. d Fraction of cells in each cancer type per EC cluster. e Fraction of EC clusters per cancer type (left) and sample origin (right). $\mathbf{f ~ N o r m a l / t u m o r ~ r a t i o ~ o f ~ r e l a t i v e ~ p e r c e n t a g e ~ o f ~ E C ~ c l u s t e r s , ~}<1$ indicates tumor enrichment. Tip ECs $\left(\mathrm{FDR}=1.4 \times 10^{-141}\right)$ and HEVs $\left(\mathrm{FDR}=2.3 \times 10^{-60}\right)$ were enriched in tumor. g t-SNEs of cEC clusters by unaligned clustering, colour-coded by cluster, sample origin and cancer type, including a zoom-in of the NEC4 cluster (right). h t-SNE of marker gene expression in CEC clusters. i-k Heatmap of differentially expressed genes in CEC clusters (i), of TF activity by SCENIC for EC (j) or CEC clusters (k). I, $\mathbf{m}$ Heatmap showing metabolic activity for EC (I) or CEC clusters (m).

Metabolic pathway analysis revealed distinct metabolic signatures among EC phenotypes (Fig. 2l, m): glycolysis and oxidative phosphorylation, which promote vessel sprouting, ${ }^{19}$ were upregulated in tip cells, while fatty acid oxidation, essential for lymphangiogenesis was increased in lymphatic ECs. ${ }^{19}$ Metabolic activities within $\mathrm{CECs}$ also differed: carbonic acid metabolism was most active in C3_NEC1, confirming these are alveolar CECs, which actively convert carbonic acid into $\mathrm{CO}_{2}$ during respiration. However, carbonic acid metabolism was reduced in C3_TECs, which instead deployed glycolysis and oxidative phosphorylation (Supplementary information, Fig. S2j). Similar characteristics were observed when assessing activation of cancer hallmark pathways (Supplementary information, Fig. S2k, I).
Fibroblasts show the highest cancer type specificity

Fibroblasts are highly versatile cell types endowed with extensive heterogeneity. ${ }^{20}$ Indeed, unaligned clustering of 24,622 fibroblasts resulted in 17 clusters (Fig. 3a, b), which were often tissue-specific (Supplementary information, Fig. S3a-d). Particularly, C1-C3 represented colon-specific clusters derived from normal tissue, while C4-C6 represented stroma (C4, C5) and mesotheliumderived cells (C6) specific for the ovary. C1-C6 fibroblasts were excluded from CCA, because they have a tissue-specific identity, localization and function that are unlikely to have counterparts in other tissues (see below). All other fibroblasts clustered into 5 clusters shared across cancer types and patients (C7-C11; Fig. 3c-e; Supplementary information, Fig. S3e). Three other CCA clusters 


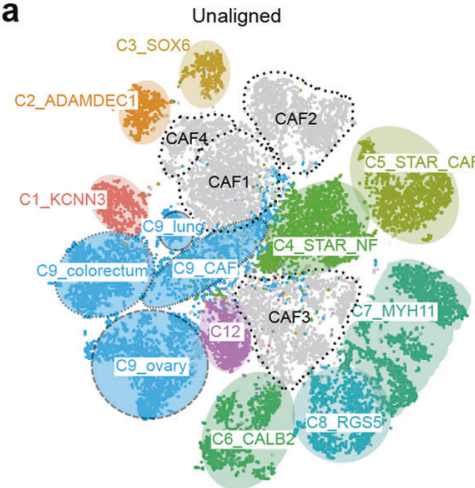

d

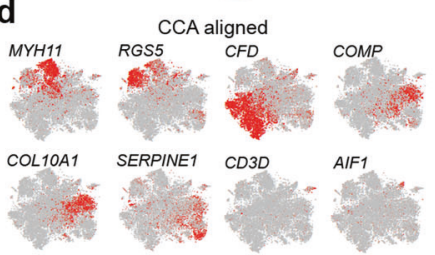

h
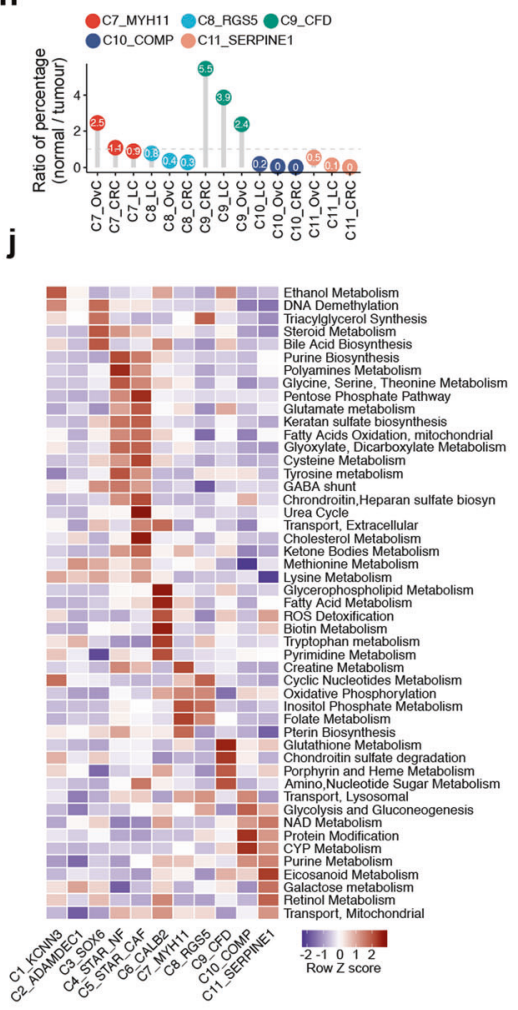

b

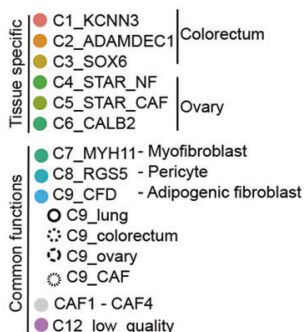

e

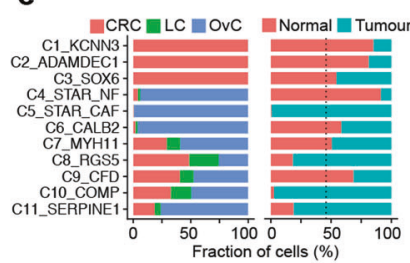

i

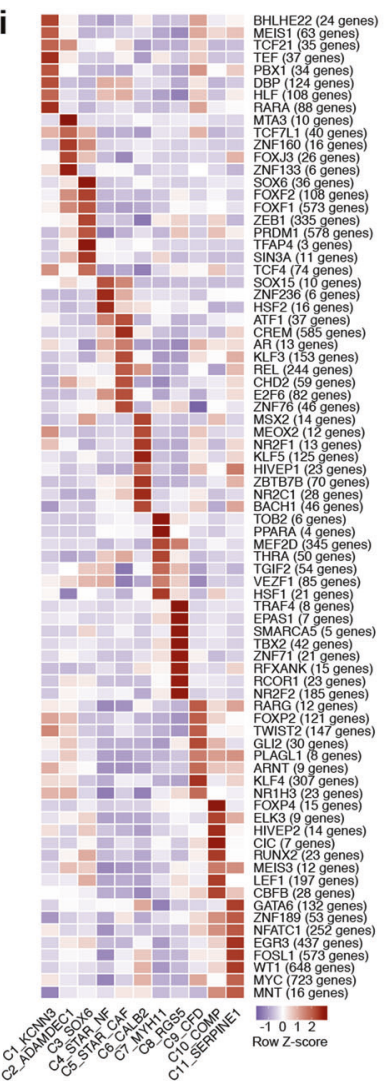

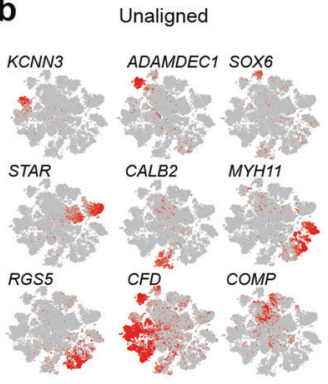

C

CCA aligned

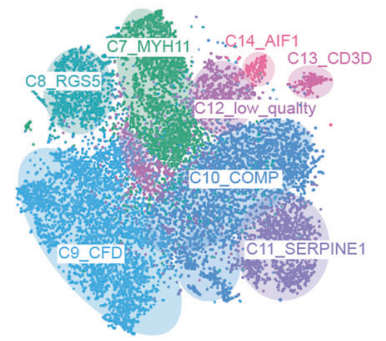

D. C7_MYH11 - Myofibroblast

- C8_RGS5 - Pericyte

- C9_CFD - Adipogen

C11_SERPINE1|CAF

- C12_low_quality

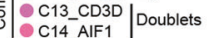

\section{g}

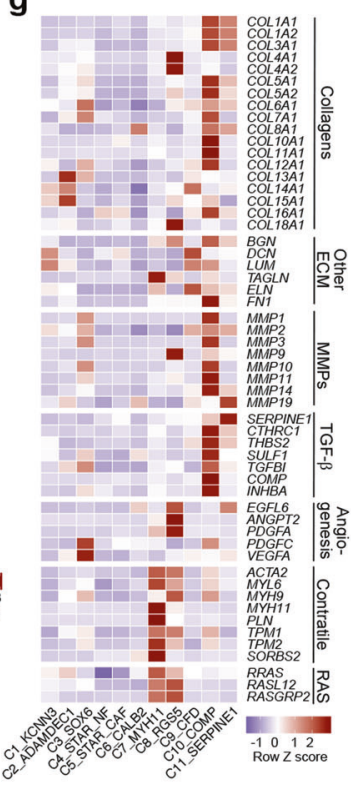

Fig. 3 Characterization of $\mathbf{2 4 , 6 2 2}$ fibroblasts. a t-SNE colour-coded for annotated fibroblasts by unaligned clustering. $\mathbf{b}$ t-SNEs with marker gene expression in unaligned clusters. $\mathbf{c}$ t-SNE colour-coded for annotated fibroblasts by CCA. d t-SNE with marker gene expression in CCA clusters. e Fraction of fibroblast clusters per cancer type (left) and sample origin (right). C7-C11s are shared by CRC, LC and OvC. f, $\mathbf{g}$ Heatmap of marker gene expression (f) and functional gene sets $(\mathbf{g})$. $\mathbf{h}$ Normal/tumor ratio of relative percentage of fibroblast clusters, $<1$ indicates tumor enrichment. Pericytes were enriched in tumor $\left(F D R=7.8 \times 10^{-10}\right)$. $\mathbf{i}$, j Heatmap of TF activity (i) or metabolic activity (j) in fibroblast clusters.

represented a low-quality (C12) or doublet cluster (C13_CD3D, C14_AIF1) (Supplementary information, Fig. S3f, g). Fibroblasts therefore consist of 11 cellular phenotypes: tissue-specific clusters C1-C6 identified by unaligned clustering and shared clusters C7-C11 identified by CCA (Fig. 3f, $g$ for marker genes and functional gene sets).

Colon-specific C1-C3s mostly resided in normal tissue (Fig. 3e). C1_KCNN3 fibroblasts co-expressed KCNN3 and P2RY1 (Fig. 3f), a potassium calcium-activated channel (SK3-type) and purine receptor (P2Y1), respectively. Their co-expression defines a novel excitable cell that co-localizes with motor neurons in the gastrointestinal tract and regulates their purinergic inhibitory response to smooth muscle function in the colon. ${ }^{21,22} \mathrm{C} 1$ KCNN3s also expressed $L Y 6 H$, a neuron-specific regulator of nicotineinduced glutamatergic signalling, ${ }^{23}$ suggesting these cells to regulate multiple neuromuscular transmission processes. 
750

C2_ADAMDEC1s represented mesenchymal cells of the colon lamina propria, ${ }^{24}$ characterised by $A D A M D E C 1$ and $A P O E$. C3_SOX6s were marked by SOX6 expression, as well as BMP4, $B M P 5, W N T 5 A$ and FRZB expression (Fig. 3f). They located in close proximity to the epithelial stem cell niche and promote stem cell maintenance in the colon. ${ }^{24}$ C4-C5 ovarian stroma cells were marked by STAR and FOXL2, ${ }^{25,26}$ which promote folliculogenesis. ${ }^{27}$ Both clusters also expressed $D L K 1$, which is typical for embryonic fibroblasts. C4_STARs were derived from normal tissue, while C5_STARs were exclusive to tumor tissue, suggesting that C4_STARs give rise to C5_STARs. ${ }^{25}$ Based on calretinin (CALB2) and mesothelin (MSLN) expression, C6_CALB2s were likely to represent mesothelium-derived cells. ${ }^{28}$ These cells were especially enriched in omentum (Supplementary information, Fig. S3h), known to contain numerous mesothelial cells.

C7_MYH11 corresponded to myofibroblasts and were characterised by high expression of smooth muscle-related contractile genes, including MYH11, PLN and ACTG2 (Fig. 3f). C8_RGS5 represented pericytes (RGS5, PDGFRB), which similar as myofibroblasts expressed contractile genes, but also showed pronounced expression of RAS superfamily members (RRAS, RASL12). Additionally, pericytes expressed a distinct subset of collagens (COL4A1, COL4A2, COL18A1), genes involved in angiogenesis (EGFL6, ANGPT2; Fig. $3 \mathrm{~g}$ ) and vessel maturation (NID1, LAMA4, NOTCH3; Supplementary information, Fig. S3i). Pericytes were enriched in malignant tissue (Fig. 3e, h; Supplementary information, Fig. S3j). When comparing pericytes from malignant versus normal tissue, the former exhibited increased expression of collagens and angiogenic factors (PDGFA, VEGFA; Supplementary information, Fig. S3k), but reduced expression of the vascular stabilization factor TIMP3. ${ }^{29}$ These differences may contribute to a leaky tumor vasculature. C9_CFDs expressed adipocyte markers adipsin (CFD) and apolipoprotein $D(A P O D)$, suggesting these are adipogenic fibroblasts. They are positively associated with aging in the dermis, ${ }^{30}$ but their role in malignancy has not been established. Notably, in the unaligned clusters, C9s separated into 3 tissuespecific clusters and a single cancer-associated fibroblasts (CAF) cluster (Fig. 3a), suggesting that C9 fibroblasts (similar as CECs) lose tissue-specificity in the TME.

C10-C11 represented CAFs showing strong activation of cancer hallmark pathways, including glycolysis, hypoxia, and epithelial-tomesenchymal transition (Supplementary information, Fig. S3I). C10_COMPs typically expressed metalloproteinases (MMPs), TGF $\beta$ signalling molecules and extracellular matrix (ECM) genes, including collagens (Fig. $3 \mathrm{~g}$ ). They also expressed the TGF- $\beta$ coactivator COMP, which is activated during chondrocyte differentiation, and activin (INHBA), which synergizes with TGF- $\beta$ signalling. ${ }^{31,32}$ Accordingly, chondrocyte-specific TGF- $\beta$ targets (COL10A1, COL11A1) were strongly upregulated. C11_SERPINE1s exhibited increased expression of SERPINE1, IGF1, WT1 and CLDN1, which all promote cell migration and/or wound healing via various mechanisms. ${ }^{33-36}$ They also expressed collagens, albeit to a lesser extent as C10_COMPs. Additionally, high expression of the proangiogenic EGFL6 suggests these cells to exert paracrine functions. $^{37,38}$ Interestingly, the number of C10-C11 CAFs positively correlated with the presence of cancer cells (Supplementary information, Fig. S3m), confirming the role of CAFs in promoting tumor growth. ${ }^{20}$

Using SCENIC, we identified TFs unique to each fibroblast cluster (Fig. 3i). For instance, MYC and EGR3 underpinned C11_CAFs, while pericytes were characterised by EPAS1, TBX2 and NR2F2 activity. Interestingly, MYC activation of CAFs promotes aggressive features in cancer cells through upregulation of unshielded RNA in exosomes. ${ }^{39}$ At the metabolic level, we observed that creatine and cyclic nucleotide metabolism, which are essential for smooth muscle function, were upregulated in myofibroblasts (C7), while glycolysis was most prominent in C10-C11 CAFs (Fig. 3j). Indeed, highly proliferative CAFs rely on aerobic glycolysis and their glycolytic adaptation promotes a reciprocal metabolic symbiosis between CAFs and cancer cells. ${ }^{20}$

Dendritic cells, novel markers of CDC maturation revealed Clustering the transcriptomes of 2722 DCs identified 5 different DC phenotypes using unaligned and CCA-aligned approaches (Fig. 4a). $92 \%$ of cells clustered similarly with both approaches, suggesting DCs in line with their non-resident nature to have limited cancer type specificity. C1_CLEC9As corresponded to conventional DCs type 1 (CDC1; CLEC9A, XCR1) ${ }^{40,41}$ C2_CLEC10As to $\mathrm{CDC}$ s type 2 (CDC2; CD1C, CLEC10A, SIRPA), while C3_CCR7s represented migratory CDCs (CCR7, CCL17, CCL19; Fig. 4b, c; Supplementary information, Fig. S4a, b). Further, C4_LILRA4s represented plasmacytoid DCs (pDCs; LILRA4, CXCR3, IRF7), while C5_CD207s were related to CDC2s based on CD1C expression. C5_CD207s additionally expressed Langerhans cell-specific markers: CD207 (langerin) and CD1A, but not the epithelial markers CDH1 and EPCAM, typically expressed in Langerhans cells. ${ }^{42}$ These cells therefore likely represented a subset of $\mathrm{CDC} 2 \mathrm{~s}$ with a similar expression as Langerhans cells. Notably, Langerhans-like and migratory DCs were not previously characterised by scRNA-seq, possibly because these studies focused on blood-derived DCs. ${ }^{40}$

Overall, C2_CLEC10As were most abundant, while the number of other DCs varied per cancer type. For instance, C3 was rare in $\mathrm{OvC}$, and $\mathrm{C} 5$ enriched in malignant tissue (Fig. 4d, e; Supplementary information, Fig. S4c, d). SCENIC confirmed known TFs to underlie each DC phenotype, including BATF3 for CDC1s, CEBPB for CDC2s, NFKB2 for migratory CDCs and TCF4 for pDCs (Fig. 4f, g). We also identified novel TFs (Supplementary information, Table S8). For instance, SPI1, a master regulator of Langerhans cell differentiation, ${ }^{43}$ and $R X R A$, required for cell survival and antigen presentation in Langerhans cells, ${ }^{44}$ were both expressed in C5. Cancer hallmark pathway analysis revealed activation of interferon- $\alpha$ and $-\gamma$ signalling in migratory $C D C s$, while metabolic pathway analysis confirmed a critical role for folate metabolism (Supplementary information, Fig. S4e, f). ${ }^{45}$

By leveraging trajectory inference analyses (using 3 different pipelines; see Materials and methods), we recapitulated the CDC maturation process and observed that $\mathrm{CDC} 2 \mathrm{~s}$ are enriched in the migrating branch (Fig. 4h, i), suggesting that migratory cDCs originate from $\mathrm{CDC} 2 \mathrm{~s}$ but not $\mathrm{CDC} 1 \mathrm{~s}$, at least in tumors. Consistent herewith, some migratory CDC-related genes, i.e., CCL17 and $C C L 22$, were already upregulated in a subset of CDC2s (Supplementary information, Fig. $\mathrm{S4g}$ ), highlighting that $\mathrm{CDC} 2 \mathrm{~s}$ are in a transitional state. In contrast, CDC maturation markers CCR7 and $\angle A M P 3$ were only upregulated at a later stage of the trajectory (Fig. 4j; Supplementary information, Fig. S4h). ${ }^{46}$ Interestingly, in OvC, CDC2s got stuck early in the differentiation lineage compared to CRC and LC (Supplementary information, Fig. S4i). By modelling expression along the branches, we retrieved 4 clusters with distinct temporal expression (Fig. 4k), in which we identified 30 and 210 genes up- or down-regulated (Supplementary information, Table S9). For example, CLEC10A was gradually lost during CDC2 maturation, while $B I R C 3$ was upregulated, suggesting they represent novel markers of $\mathrm{CDC}$ maturation. Also, when investigating TF dynamics from $C D C 2 s$ to migratory $C D C s$, we identified 22 up- and 23 down-regulated TFs, respectively (Fig. 4l; Supplementary information, Fig. S4j).

B-cells, comprehensive taxonomy and developmental trajectory Amongst the 15,247 B-cells, we identified 8 clusters using unaligned clustering (Fig. 5a). Three of these represented follicular B-cells (MS4A1/CD20), which reside in lymphoid follicles of intratumor tertiary lymphoid structures, while 4 clusters were antibodysecreting plasma cells (MZB1 and SDC1/CD138) (Supplementary information, Fig. S5a, b). We also retrieved a T-cell (C9_CD3D) doublet cluster (Supplementary information, Fig. S5c). CCA identified 2 additional clusters: one unaligned follicular B-cell 
a

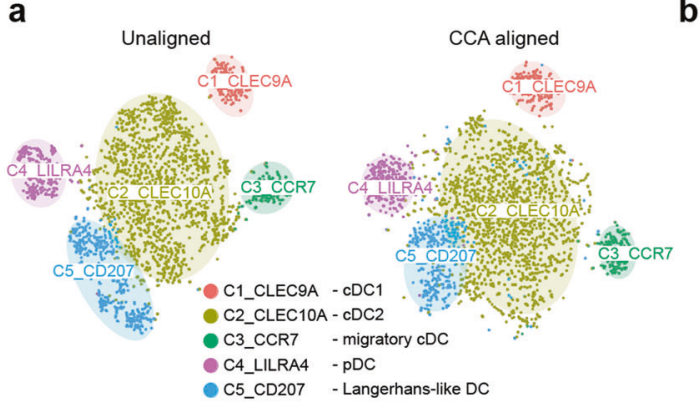

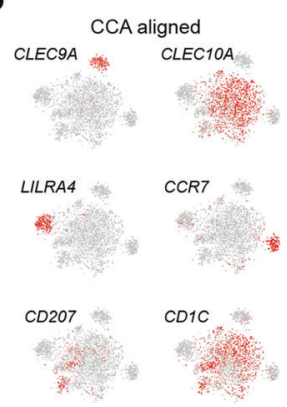

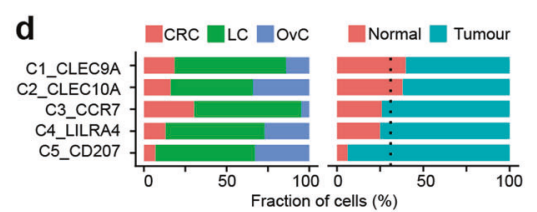

g

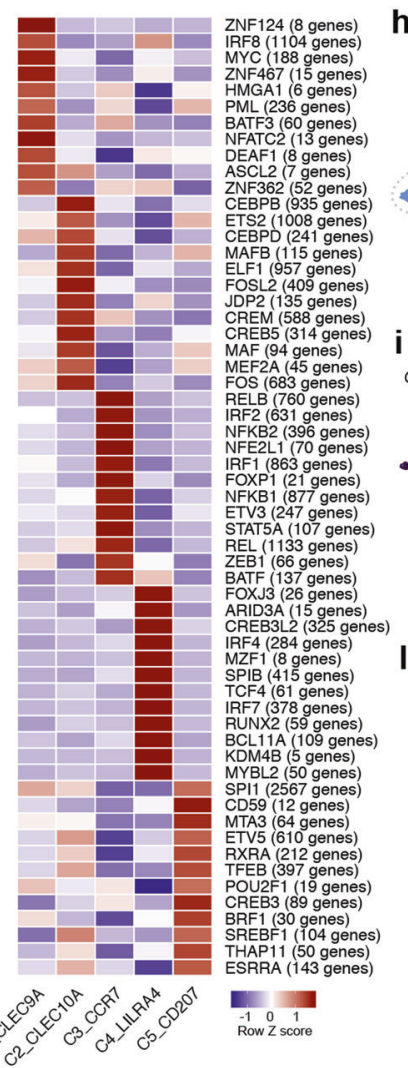

e

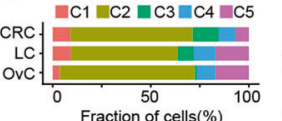

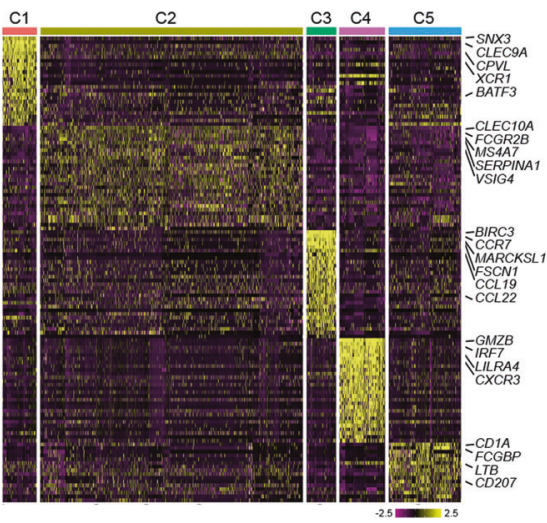

\section{f}
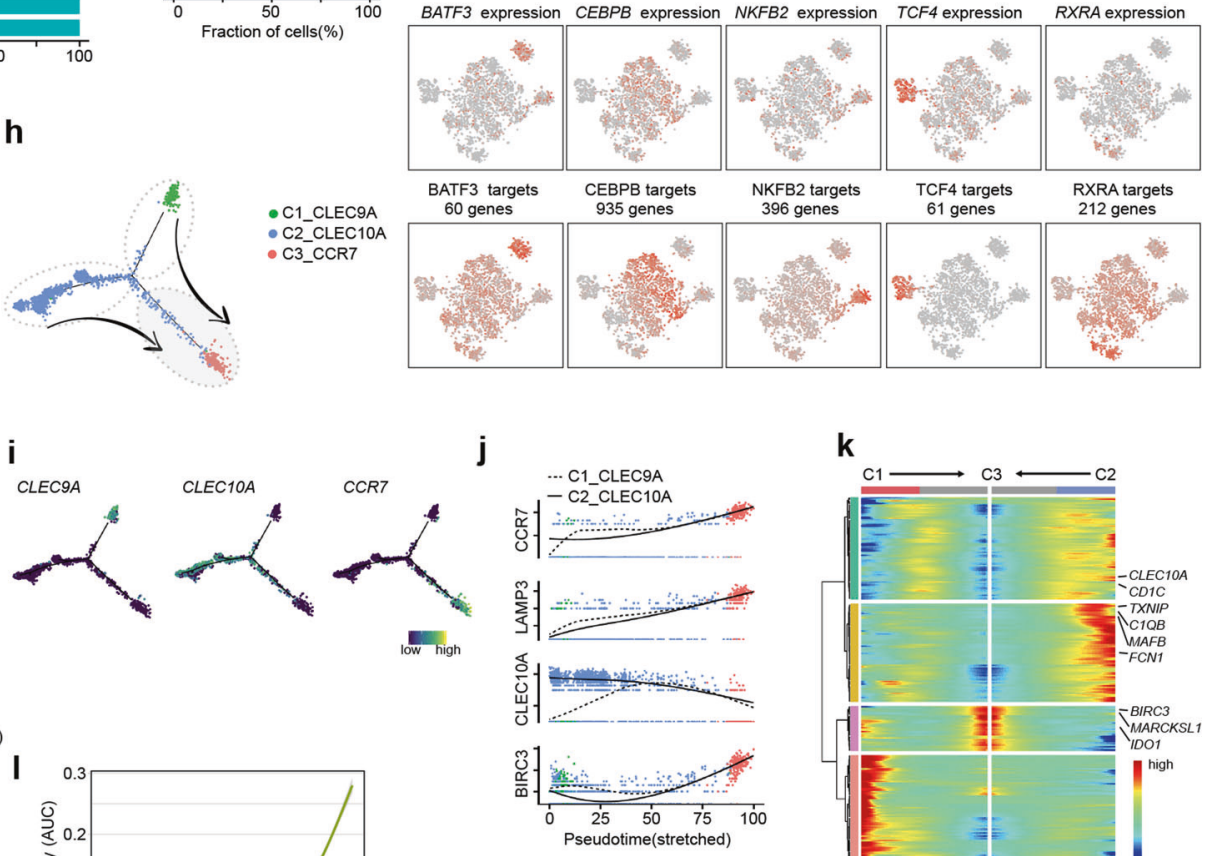

k

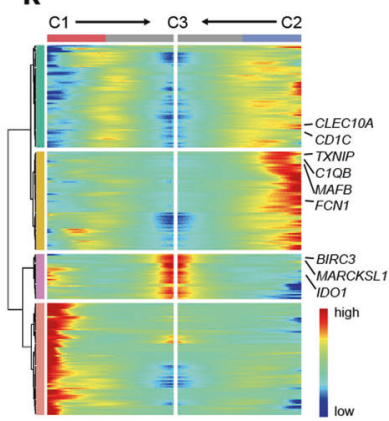

Fig. 4 Clustering 2722 DCs. a t-SNEs colour-coded for annotated DCs by unaligned and CCA-aligned clustering. $\mathbf{b}$ t-SNEs with DC marker gene expression in CCA aligned clusters. c Heatmap for differential gene expression in unaligned clusters. $\mathbf{d}$ Fraction of DC clusters per cancer type (left) and sample origin (right). Migratory CDCs were depleted in OvC (FDR $=0.017$ ). e Fraction of cells in each cancer type per cluster. $\mathrm{f} t-$ SNEs with gene expression (upper) and corresponding TF activity (lower). $\mathbf{g}$ Heatmap showing TF activity in CCA-aligned clusters. $\mathbf{h}$ Trajectory inference analysis of CDC-related subclusters. i Marker gene expression along the CDC trajectory. $\mathbf{j}$, $\mathbf{k}$ Marker gene expression (j) and expression dynamics (k) during CDC maturation. I TF activation dynamics of CDC2 to migratory CDC differentiation.

cluster, which was split into 2 separate clusters (C2 and C3; Fig. 5a, b), and one additional cancer cell (C10_KRT8) doublet cluster (Supplementary information, S5c). Overall, this resulted in 8 relevant B-cell clusters, each of them characterised by functional gene sets (Fig. 5c).

Follicular B-cells were composed of mature-naïve $\left(C D 27^{-}, \mathrm{C} 1\right)$ and memory $\left(C D 27^{+}, C 2-C 4\right)$ B-cells (Fig. 5c). The former cells are characterised by a unique $C D 27^{-} / I G H D^{+}(\operatorname{lgD}) / / G H M^{+}(\lg M)$ signature and give rise to the latter by migrating through the germinal centre (GC; referred to as GC-memory B-cells). This process requires expression of migratory factors $C C R 7$ (for GC entry) and GPR183 (for GC exit; Supplementary information, Fig. S5d). ${ }^{47}$ In the GC, IGHM undergoes class-switch recombination to form other immunoglobulin isotypes. Indeed, GC-memory B-cells separated into $I G H M^{+}$and $I G H M^{-}$populations, i.e., $\mathrm{C} 2 I_{G H M^{+}}$and $\mathrm{C} 3 \mathrm{IGHM}^{-}$ clusters (Fig. $5 \mathrm{a}-\mathrm{C}$ ). A rare population of memory B-cells is generated independently of the GC. ${ }^{48}$ These GC-independent memory B-cells corresponded to $\mathrm{C} 4$ _CD $27^{+} / \mathrm{CD} 38^{+}$s, lacking GC migratory factors GPR183 and CCR7, but expressing the anti-GC migration factor RGS13, which may form the basis for their GC 


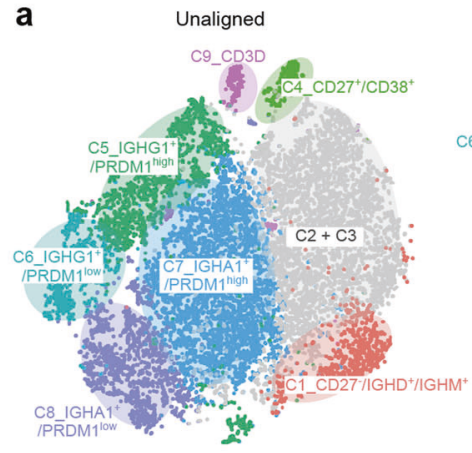

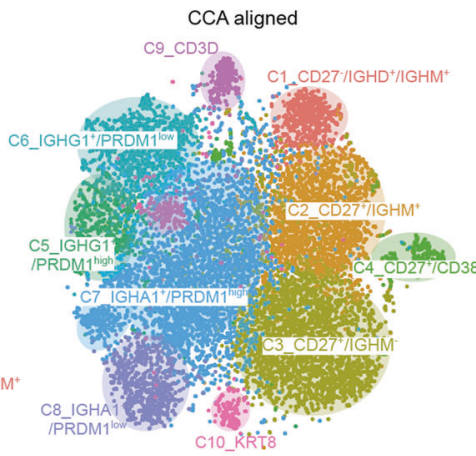

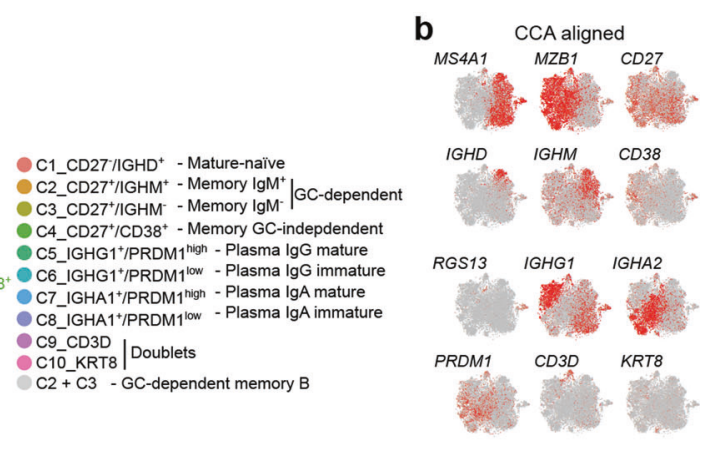

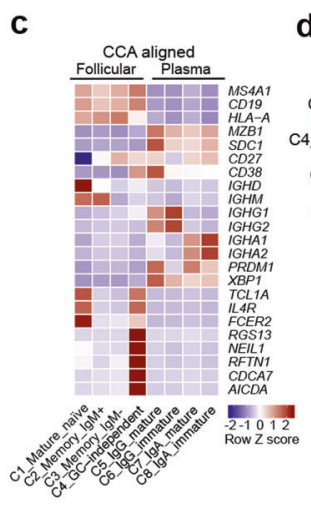

d
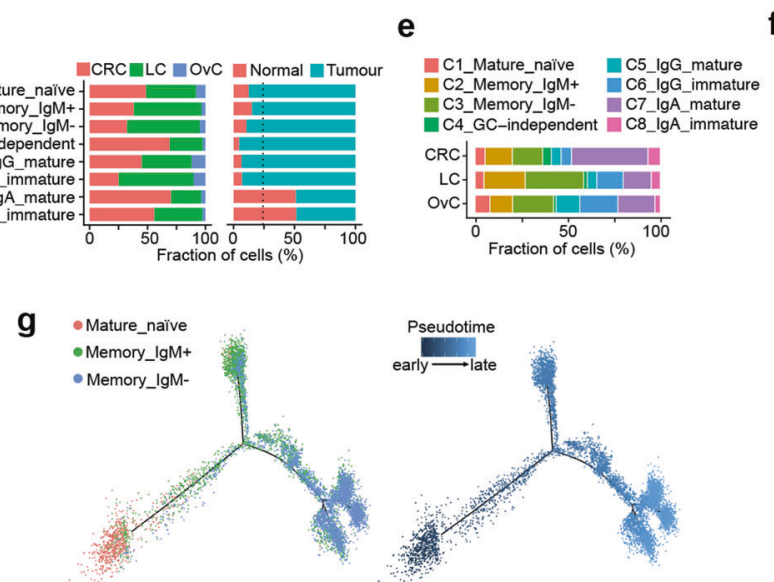

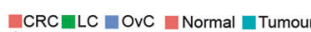

C1 Mature naive

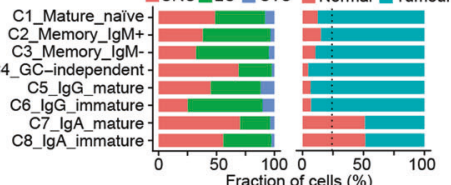

C1_Mature_naïve C5_lgG_mature C3 Memory lgMC4 GC-independent C8 IgA immatu LC.
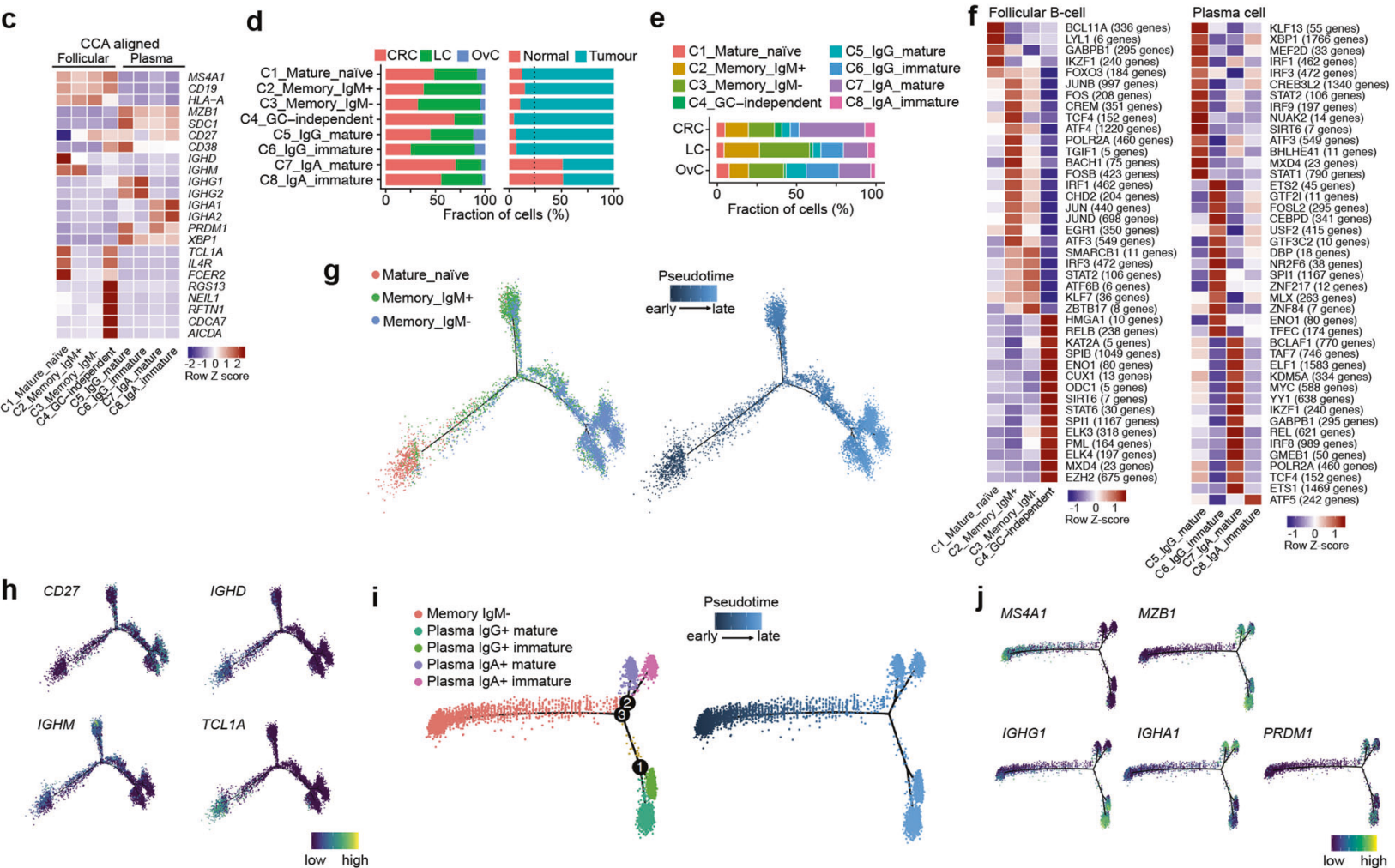

Fig. 5 B-cell taxonomy and developmental trajectory. a t-SNEs colour-coded for annotated B-cells using unaligned and CCA-aligned clustering. b t-SNEs with marker gene expression in CCA clusters. $\mathbf{c}$ Heatmap of functional gene sets in CCA clusters. d Fraction of B-cell clusters per cancer type (left) and sample origin (right). e Fraction of cells in each cancer type per cluster. f Heatmap with TF activity by SCENIC, for follicular B-cell (left) or plasma cell clusters (right). g Developmental trajectory for GC-dependent memory B-cells, colour-coded by cell type (left) and pseudotime (right). $\mathbf{h}$ Marker gene expression of the GC-memory B-cell trajectory as in $\mathbf{g}$. $\mathbf{i}$ Trajectory of IgM ${ }^{-}$memory $^{B}$ to lgG ${ }^{+}$or $\operatorname{lgA}^{+}$plasma cells, colour-coded by branch type (left) and pseudotime (right). $\mathbf{j}$ Marker gene expression dynamics during plasma cell differentiation as in i.

exclusion $^{49}$ (Fig. 5b; Supplementary information, Fig. S5d). Although little is known about GC-independent B-cells, they appear early during immune response and respond to a broader range of antigens with less specificity as GC-memory B-cells. ${ }^{50}$ Interestingly, $\mathrm{C} 4 \mathrm{~s}$ exhibited an expression signature intermediate to mature-naïve and GC-memory B-cells (Supplementary information, Fig. S5e). Expression of IGHD and IGHM was low, while IGHG1 and IGHG3 were elevated (Supplementary information, Fig. S5f), suggesting $\mathrm{C} 4 \mathrm{~s}$ to have completed class-switch recombination. Indeed, AICDA expression, which induces mutations in class-switch regions during recombination, ${ }^{50}$ was elevated in C4s (Fig. 5c). They were also characterised by several uniquely expressed genes and enriched for proliferative cells (Supplementary information, Fig. S5g and Table S5). Next to follicular B-cells, we identified 4 clusters of plasma B-cells (C5-C8), which can be separated based on expression of immunoglobulin heavy chains, i.e., IGHG1 (IgG) versus IGHA1 $(\operatorname{Ig} \mathrm{A})$. Both could be further stratified based on their antibody-secreting capacity as determined by PRDM1 (Blimp-1) ${ }^{50}$ : low versus high for immature versus mature plasma cells, overall resulting in 4 plasma B-cell clusters (Fig. 5c).

Importantly, B-cell clusters were enriched in all tumors, except for IgA-expressing plasma cells, which mainly resided in mucosarich normal colon (Fig. 5d, e; Supplementary information, Fig. S5h-j). Additionally, GC-independent memory B-cells were most prevalent in CRC. B-cells were also enriched in border versus core fractions of LC tumors (Supplementary information, Fig. S5k). Using SCENIC, each B-cell cluster was characterised by a unique set of TFs (Fig. 5f). For instance, GC-independent memory B-cells upregulated NF-KB (RELB) and STAT6, which is known to suppress GPR183. ${ }^{51}$ Some TFs were upregulated in mature (PRDM $1^{\text {high }}$ ) plasma cells, irrespective of their heavy chain expression. These included multiple immediate-early response TFs (FOS, JUND and 
EGR1) and the interferon regulatory factor IRF1 (Supplementary information, Fig. S5I), suggesting they are involved in plasma cell maturation. C5_lgG_mature B-cells, relative to all other plasma Bcells, exhibited strong activation of nearly all cancer hallmark pathways, indicating an active role of $\mathrm{C} 5 \mathrm{~s}$ in the TME (Supplementary information, Fig. S5m).

Trajectory inference analysis confirmed that mature-naïve Bcells differentiate into either $\mathrm{GC}-$ memory $\operatorname{lgM}^{+}$or $\operatorname{lgM}^{-}$branches. As expected, $\operatorname{lgM}^{+}$but not $\operatorname{lgM}^{-}$cells were located halfway the trajectory (Fig. 5g; Supplementary information, Fig. S5n), confirming $\operatorname{lgM}^{+}$cells to undergo class-switch recombination into $\lg ^{-}$ cells. Memory B-cells of the $\operatorname{lgM}^{+}$and $\operatorname{lgM}^{-}$lineages were similarly distributed in OvC and CRC, but in LC they were more differentiated (Supplementary information, Fig. S5o). By overlaying gene expression dynamics on the trajectory, we identified several up- or down-regulated genes along the pseudotime, including CD27 and TCL1A, respectively (Fig. 5h; Supplementary information, Fig. S5p, q and Table S9). In line with CCR7 and GPR183 determining GC entry and exit, CCR7 was expressed in maturenaïve B-cells (C1, before entry) but disappeared in $I G H M^{-}$B-cells. Vice versa, GPR183 was only expressed after GC entry (C2 and C3, Supplementary information, Fig. S5d, q). Similarly, we assessed the trajectory of class-switched GC-memory B-cells (C3) differentiating into plasma cells. We confirmed that GC-memory B-cells differentiate into either $\operatorname{lgG}^{+}$- or $\lg A^{+}$-expressing plasma cells (Fig. 5i), and that both branches subsequently dichotomize into mature or immature states based on PRDM1 expression (Fig. 5j). Cells were similarly distributed along the trajectory regardless of the cancer type, although in LC there was an enrichment towards the beginning of the IgA lineage (Supplementary information, Fig. S5r). Further, when assessing underlying expression dynamics along the trajectory, we identified several genes staging the differentiation process (Supplementary information, Fig. S5s and Table S9). For example, we found TNFRSF17 (also known as B-cell maturation antigen) to increase along the $\lg \mathrm{A}^{+}$plasma cell trajectory. ${ }^{52}$

T-/NK-cells show cancer type-dependent prevalence

Altogether, 52,494 T- and natural killer (NK) cells clustered into 12 and 11 clusters using unaligned and CCA-aligned methods (Fig. 6a, b). The additional cluster identified by unaligned clustering (C12) was composed of cells from normal lung tissue (Supplementary information, Fig. S6a, b). CCA did not affect clustering of T-/NKcells in tumors, indicating that T-cells have limited cancer typespecific differences. Besides C12 and a low-quality cluster (C11, Supplementary information, Fig. S6c, d), T-/NK-cells consisted of 10 phenotypes, including $4 \mathrm{CD}^{+}{ }^{+}$T-cell $(\mathrm{C} 1-\mathrm{C} 4), 4 \mathrm{CD}^{+}{ }^{+}$-cell (C5-C8) and 2 NK-cell clusters (C9-C10).

The C1_CD8 HAVCR2 cluster consisted of exhausted CD8 ${ }^{+}$ cytotoxic T-cells characterised by cytotoxic effectors (GZMB, GNLY, IFNG) and inhibitory markers (HAVCR2, PDCD1, CTLA4, LAG3, TIGIT; Fig. 6c). C2_CD8_GZMKs represented pre-effector cells as expression of $G Z M K$ was high, but expression of cytotoxic effectors low. C3_CD8_ZNF683s constituted memory $\mathrm{CD}^{+}$T-cells based on ZNF683 expression, ${ }^{53}$ while C4_CD8_CX3CR1s corresponded to effector T-cells due to high cytotoxic marker expression. Remarkably, C4s also expressed markers typically observed in NK-cells (KLRD1, FGFBP2, CX3CR1), suggesting they are endowed with NK T-cell (NKT) activity. Similarly, based on marker gene expression, we assigned C5_CD4_CCR7s to naïve (CCR7, SELL, LEF1), C6_CD4_GZMAs to CD4 ${ }^{+}$memory/effector (GZMA, ANXA1) and C7_CD4_CXCL13s to exhausted CD4 ${ }^{+}$effector T-cells (CXCL13, PDCD1, CTLA4, BTLA). Based on the expression of FOXP3, C8_FOXP3s were assigned $\mathrm{CD}^{+}{ }^{+}$regulatory T-cells (Tregs). Finally, two clusters contained NK-cells based on NK- (NCR1, NCAM1) but not T-cell $(C D 3 D, C D 4, C D 8 A$; Fig. 6b, c) marker gene expression. Particularly, C9_NK_FGFBP2s represented cytotoxic NK-cells due to the expression of FGFBP2, FCGR3A and cytotoxic genes including
GZMB, NKG7 and PRF1, while C10_NK_XCL1s appeared to be less cytotoxic, but positive for $X C L 1$ and $X C L 2$, two chemo-attractants involved in DC recruitment enhancing immunosurveillance. ${ }^{54}$

Interestingly, T-cell clusters were highly similar to the T-cell taxonomy derived from breast, liver and lung cancer, despite underlying differences in sample preparation and single-cell technology ${ }^{33,55,56}$ (Supplementary information, Fig. S6e). Indeed, C 8 cells could be re-clustered into CLTA4 ${ }^{\text {high }}$ and CLTA4 ${ }^{\text {low }}$ clusters with corresponding marker genes ${ }^{53,56}$ (Supplementary information, Fig. S6f, g), while also both NK clusters corresponded to recently identified NK subclusters shared across organs and species. $^{57}$

Several T-cell phenotypes, especially those with inhibitory markers, were enriched in tumor tissue (Fig. 6d, e; Supplementary information, Fig. S6h). C9_NK_FGFBP2s were more prevalent in normal tissue, suggesting these to represent tissue-patrolling phenotypes of NK-cells. All T-cell clusters were more frequent in LC, while cytotoxic T-cells were rare in CRC and regulatory T-cells underrepresented in OvC (Fig. 6f). Expression of inhibitory markers (HAVCR2, LAG3, PDCD1) was enhanced in exhausted/cytotoxic C1_CD8_HAVCR2s residing in tumor versus normal tissue (Supplementary information, Fig. S6i). We also observed expression of KLRC1 (NKG2A), a novel checkpoint, ${ }^{58,59}$ exclusively in C10 NK-cells (Fig. 6C). $\mathrm{CD}^{+}$T-cell trajectory analysis revealed that $\mathrm{C} 2$ preeffector T-cells also contained naïve $\mathrm{CD}^{+} \mathrm{T}$-cells, which expressed CCR7, TFC7 and SELL, and formed the root of the trajectory (Supplementary information, Fig. S6j, k). Pre-effector T-cells then differentiated into either exhausted (C1_CD8_HAVCR2) or effector (C4_CD8_CX3CR1) T-cells (Fig. 6g). Dynamic expression of marker genes along both trajectories confirmed high expression of IFNG, inhibitory and cytotoxicity markers in the HAVCR2 trajectory (Supplementary information, Fig. S6I). Interestingly, LC CD8 ${ }^{+} \mathrm{T}$ cells were more differentiated in this trajectory and thus more exhausted compared to T-cells from CRC and OvC (Fig. 6h).

TFs underlying each T-/NK-cell phenotype were identified by SCENIC (Fig. 6i): for instance, FOXP3 was specific for C8s, as expected, while IRF9, which induces $P D C D 1,{ }^{60}$ was increased in exhausted CD8 ${ }^{+}$T-cells (C1). C1_CD8_HAVCR2 T-cells exhibited high interferon activation based on cancer hallmark analysis (Supplementary information, Fig. S6m), while metabolic pathway analysis revealed upregulation of glycolysis and nucleotide metabolism in T-cell phenotypes enriched in tumors (C1, C7-C8; Supplementary information, Fig. S6n). Finally, we noticed a negative correlation between the prevalence of cancer and immune cells, including several T-cell phenotypes (Supplementary information, Fig. S3m). When scoring cancer cells for cancer hallmark pathways and comparing these scores with stromal cell phenotype abundance, some remarkable associations were noticed. Specifically, C1_CD8_HAVCR2 T-cells were positively correlated with augmented interferon signalling, inflammation and IL6/JAK/STAT3 signalling in cancer cells (Supplementary information, Fig. S6o).

Trajectory of monocyte-to-macrophage differentiation revealed In the 32,721 myeloid cells, we identified 12 unaligned clusters, including 2 monocyte (C1-C2), 7 macrophage (C3-C9) and 1 neutrophil (C10) clusters (Fig. 7a, b). A low-quality cluster (C11) and myeloid/T-cell doublet cluster (C12_CD3D) were not discussed (Supplementary information, S7a, b). Only C8 macrophages were tissue-specific, while remaining cells clustered similarly with CCA as with unaligned clustering, expressing the same marker genes and functional gene sets (Fig. 7c; Supplementary information, Fig. S7c, d).

Monocytes clustered separately from macrophages based on reduced macrophage marker expression (CD68, MSR1, MRC1) and a phylogenetic reconstruction (Supplementary information, Fig. S7e, f). C1 CD14 monocytes represented classical monocytes based on high expression of CD14 and S100A8/9, and typically being 
a

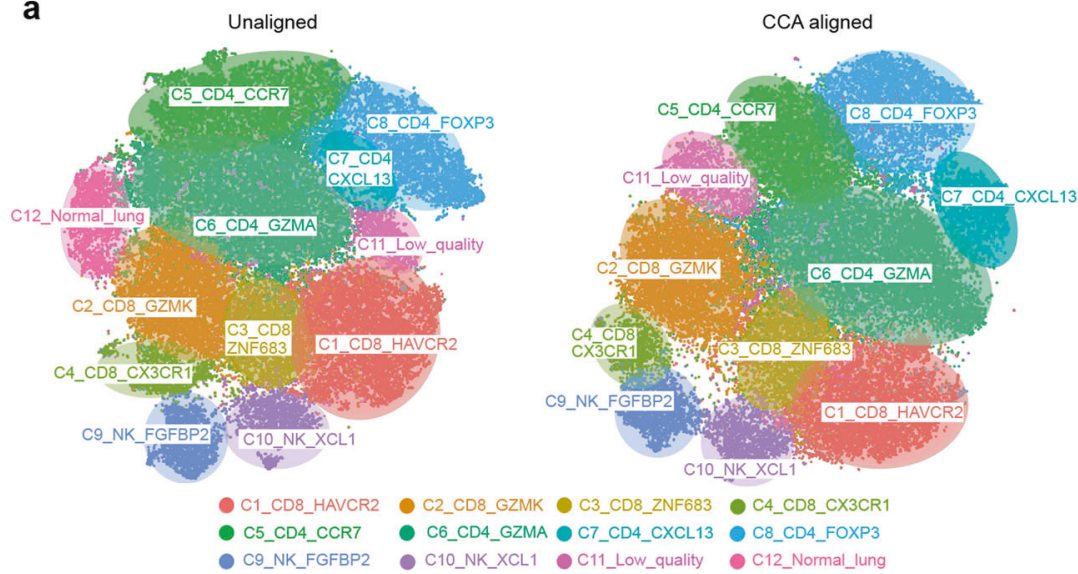

C

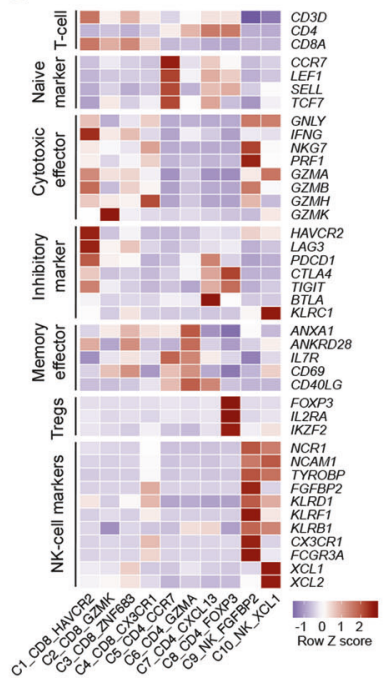

d

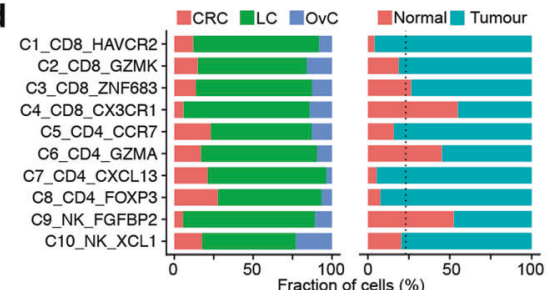

e OC1_CD8_HAVCR2 C2_CD8_GZMK OC3_CD8_ZNF683 OC4_CD8_CX3CR1 OC5_CD4_CCR7 C6_CD4_GZMA C7_CD4_CXCL13 OC8_CD4_FOXP3 C9. NK_FGFBP2 C10_NK_XCL1

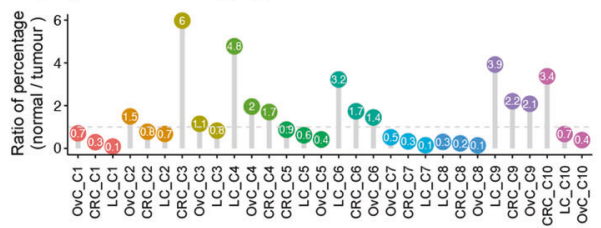

f

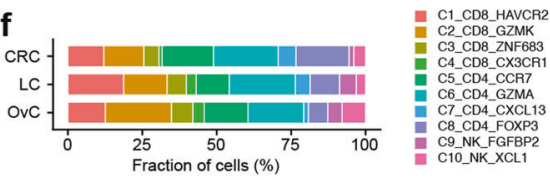

g

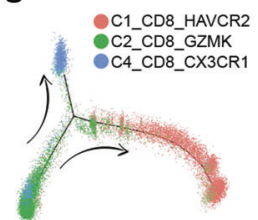

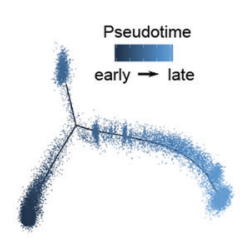

OCDB_HAVR2

h

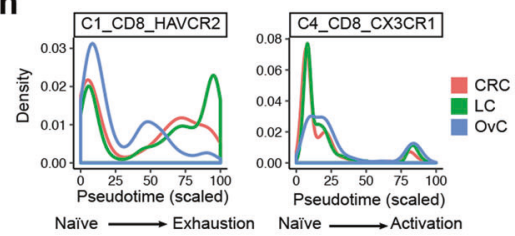

b

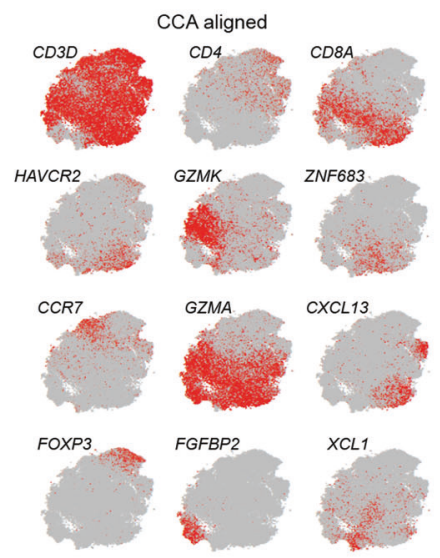

i

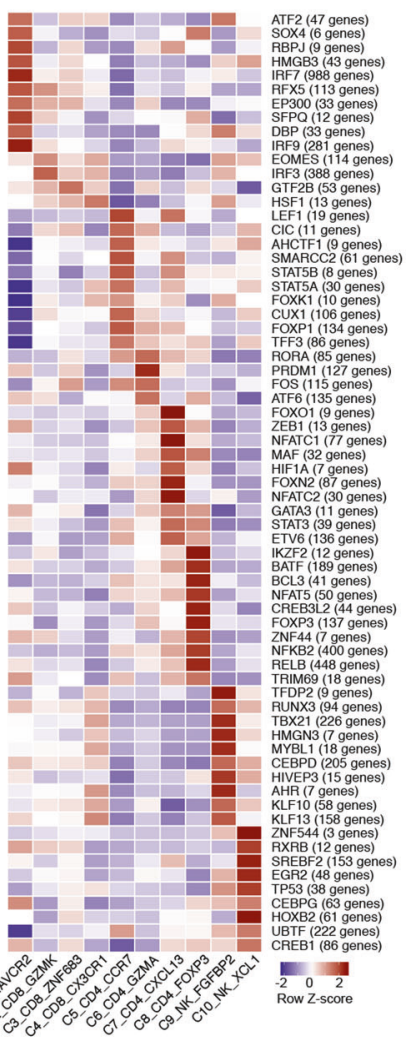

Fig. 6 Profiling 52,494 T-/NK-cells. a t-SNEs colour-coded for annotated T-/NK-cell using unaligned and CCA aligned clustering. b t-SNEs with marker gene expression in CCA clusters. c Heatmap of functional gene sets in CCA clusters. d Fraction of cells for T-/NK-cell clusters per cancer type (left) and sample origin (right). e Normal/tumor ratio of relative percentage of T-/NK-cell clusters, $<1$ indicates tumor enrichment. C1, C2, C5, C7, C8 were enriched in tumor (FDR $\left.<5.1 \times 10^{-25}\right)$, C9 was enriched in normal (FDR $=1.5 \times 10^{-219}$ ). $f$ Fraction of T-/NK-cells in each cancer type per cluster. C4 and C8 were rare in CRC (FDR $=0.019)$ and OvC (FDR $=0.034)$, respectively. $\mathbf{g}$ Heatmap with TF activity of T-/NK-cell clusters by SCENIC. $\mathbf{h}$ Differentiation trajectory for $\mathrm{CD}^{+} \mathrm{T}$ cell lineages, colour-coded by cell type (left) and pseudotime (right). $\mathrm{i}$ Density plots for CRC, LC and OvC along the two CD8 ${ }^{+}$T-cell trajectories.

recruited during inflammation. They expressed the monocyte trafficking factors SELL (CD62L) -involved in EC adhesion- and CCR2, a receptor for the pro-migratory cytokine CCL2. C2_CD16s were less abundant and represented non-classical monocytes based on low expression of CD14, but high expression of FCGR3A (CD16) and other marker genes ${ }^{61}$ (CDKN1C, MTSS1; Supplementary information, Fig. S7f). C2s constantly patrol the vasculature, express CX3CR1 (Supplementary information, Fig. S7d, g) and migrate into tissues in response to CX3CL1 derived from inflamed ECs.

Macrophages were classified based on origin (tissue-resident versus recruited) or their pro- versus anti-inflammatory role (M1like versus M2-like, Fig. 7c). C3_CCR2s and C4_CCL2s represented early-stage macrophages that were closely-related, not enriched in tumors (Fig. 7d; Supplementary information, Fig. S7e) and become replenished by classical monocytes. Specifically, C3 macrophages represented immature macrophages closely related to $C 1$ monocytes, as they also expressed CCR2 (Fig. 7b). They were characterised by pronounced M1 marker gene expression (IL1B, CXCL9, CXCL10, SOCS3; Fig. 7c). C4_CCL2s were characterised by CCL2 expression, which is another M1 marker promoting immune cell recruitment to inflammatory sites. Compared to C3s, C4 macrophages expressed less CCR2, but moderate levels of the $M 2$ marker gene $M R C 1$, suggesting an intermediate pro-inflammatory phenotype. 
a

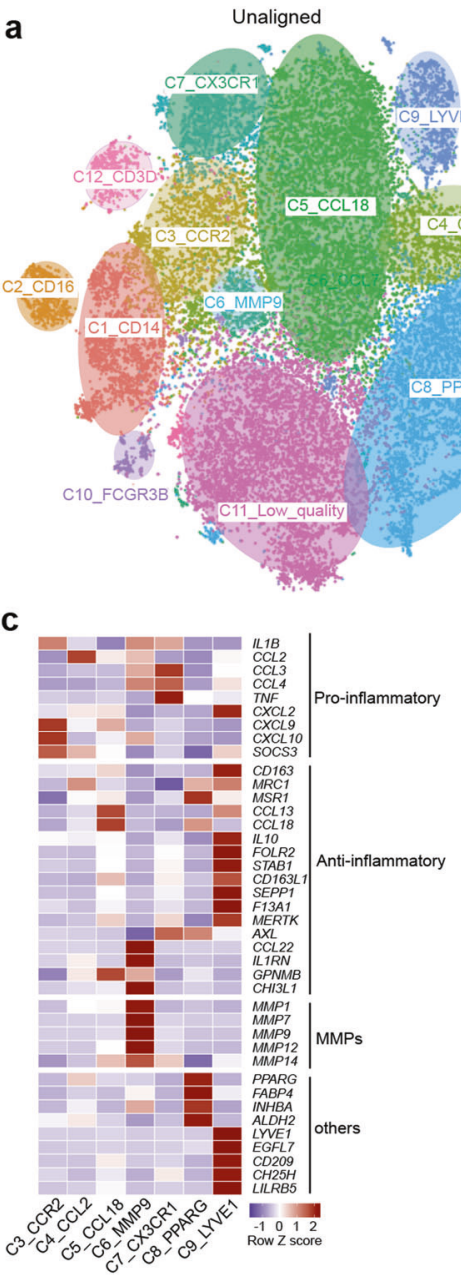

g

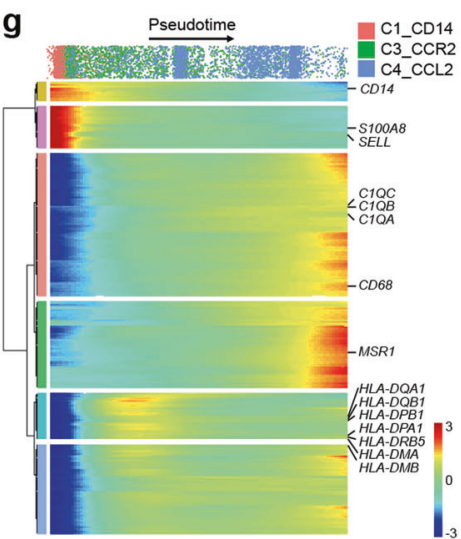

C1_CD14 Monocytes

C3_CCR2 Early stage

C4_CCL2 macrophages

C5 CCL18

Tumor-associated

C7_CX3CR1 macrophages

C8_PPARG (alveolar) Resident

C9 LYVE1 (perivascular) macrophages

C10 FCGR3B Neutrophils

C11_Low_quality

C12_CD3D Doublets

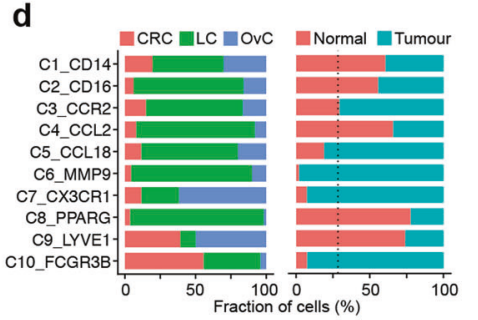

e

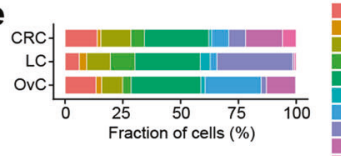

f

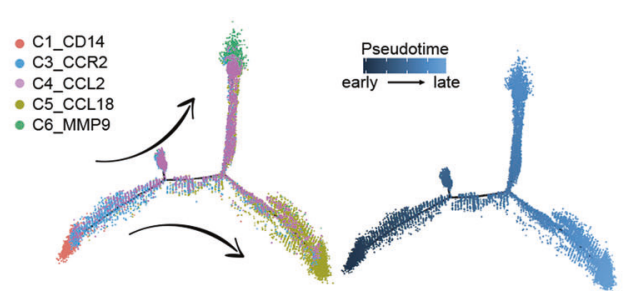

h

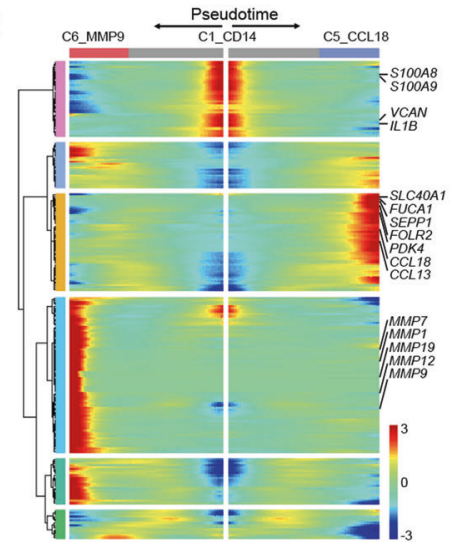

b

Unaligned

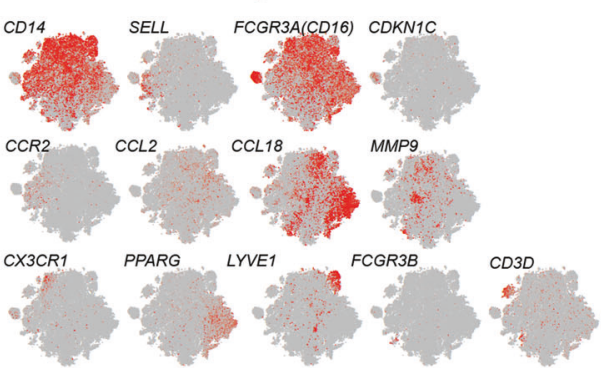

i ZNF136 7 genes)

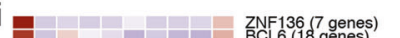

j

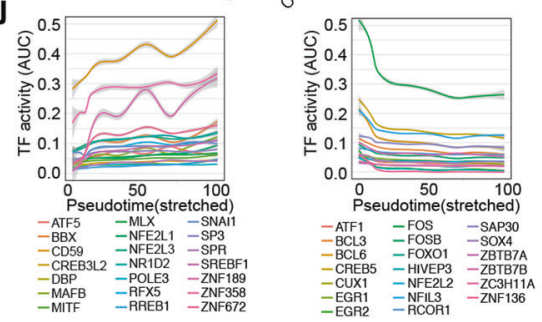

Fig. 7 Profiling of monocytes, macrophages and neutrophils. a t-SNE colour-coded for annotated myeloid cell using unaligned clustering. b t-SNEs with marker gene expression in myeloid clusters. $\mathbf{c}$ Heatmap of functional gene sets in myeloid clusters. $\mathbf{d}$ Fraction of myeloid clusters per cancer type (left) and sample origin (right). C9 was enriched in normal (FDR $=3.0 \times 10^{-31}$ ) and C8 in normal lung (FDR $\approx 0$ ) tissue. C5-C7 and $\mathrm{C} 10$ (FDR $<3.3 \times 10^{-31}$ ) were enriched in tumor. e Fraction of cells in each cancer type per cluster. $\mathbf{f}$ Monocyte-to-macrophage differentiation trajectory, colour-coded by cluster (left) or pseudotime (right). $\mathbf{g}, \mathbf{h}$ Gene expression dynamics during differentiation of C1 monocytes to C4 macrophages (g), or terminal differentiation of C5/C7 macrophages (h). i Heatmap showing TF activity by SCENIC. $\mathbf{j}$ TF activation (left) or inactivation (right) during monocyte-to-macrophage differentiation, before branching into terminal differentiation.

Macrophages belonging to $\mathrm{C} 5-\mathrm{C} 7$ clusters were enriched in malignant tissue and represented tumor-associated macrophages (TAMs, Fig. 7d; Supplementary information, Fig. S7h). C5_CCL18s represented $\sim 72 \%$ of all TAMs and were characterised by $\mathrm{M} 2$ marker expression, including CCL18 and GPNMB (Fig. 7c). Additional heterogeneity separated $\mathrm{C} 5$ cells into intermediate and more differentiated M2 macrophages, although differences were graded, consistent with a continuous phenotypic spectrum (Supplementary information, Fig. S7i). Indeed, there was more pronounced M2 marker expression (e.g., SEPP1, STAB1, CCL13) in $34 \%$ of $\mathrm{C} 5 \mathrm{~s}^{62}$ These also expressed key metabolic pathway regulators, i.e., SLC4OA1 (iron), FOLR2 (folate), FUCA1 (fucose) and PDK4 (pyruvate), linking M2 differentiation with metabolic reprogramming. C6_MMP9 macrophages expressed a unique 
756

subset of M2 markers (CCL22, IL1RN, CHI3L1) and several MMPs, suggesting a role in tumor tissue remodelling. Cancer hallmark analysis revealed enrichment in EMT, hypoxia, glycolysis and many other pathways (Supplementary information, Fig. S7j). C7_CX3CR1 macrophages expressed genes involved both in $\mathrm{M} 1$ and $\mathrm{M} 2$ polarization (CCL3, CCL4, TNF, $A X L$, respectively, Fig. 7c). Interestingly, AXL is involved in apoptotic cell clearance, ${ }^{63}$ whereas other M2 markers involved in pathogen clearance, i.e., MRC1 and CD163, were absent, suggesting a unique phagocytic pattern of $C 7$ cells. They are also correlated with poor prognosis in OvC and CRC. ${ }^{64,65}$ Of note, C7 macrophages shared their CD $16^{\text {high }} /$ CX3CR $1^{\text {high }}$ phenotype with C2 non-classical monocytes, suggesting both clusters may be related (Supplementary information, Fig. S7g). C8_PPARG macrophages corresponded to resident alveolar macrophages due to expression of the resident alveolar macrophage marker PPARG. They were exclusive to normal lung tissue (Fig. 7d), expressed established M2 markers (MSR1, CCL18, AXL) ${ }^{62,66}$ in addition to anti-inflammatory genes (FABP4, $A L D H 2){ }^{67,68}$ C9 LYVE1 macrophages also represented resident macrophages with pronounced $\mathrm{M} 2$ marker expression and enrichment in normal tissue. They often locate at the perivasculature of different tissues where they contribute to both angiogenesis and vasculature integrity. ${ }^{69-71}$ Indeed, C9 macrophages expressed the angiogenic factor EGFL7, but also immunomodulators CD209, CH25H and LILRB5, which are implicated in both innate and adaptive immunity. ${ }^{62,72,73}$

Finally, the C10_FCGR3B cluster represented neutrophils expressing the neutrophil-specific antigen CD16B (encoded by FCGR3B), but not $M P O$, which is typically expressed in neutrophils during inflammation and microbial infection. $\mathrm{C} 10$ cells expressed proinflammatory factors (CXCL8, IL1B, CCL3, CCL4; Supplementary information, Fig. $\mathrm{S7g}$ ) and, in line with their pro-tumor activity, also pro-angiogenic factors (VEGFA, PROK2). ${ }^{74}$ Notably, neutrophils were strongly enriched in malignant tissue, but were characterised by low transcriptional activity (689 detected genes/cell; Fig. 7d; Supplementary information, Fig. S7b).

Interestingly, except for resident alveolar macrophages (C8), all myeloid clusters were present in each cancer type, albeit with some preferences (Fig. 7d, e). Similar to other scRNA-seq studies, ${ }^{4,6,7,75}$ we failed to identify myeloid-derived suppressor cells (MDSCs), which are known to be morphologically and phenotypically similar to monocytes and neutrophils. Indeed, the S100A8/9 markers expressed in MDSCs were also highly expressed in $\mathrm{C} 1$ monocytes and $\mathrm{C} 10$ neutrophils ${ }^{76-78}$ (Supplementary information, Fig. S7g). This highlights that scRNA-seq fails to fully dissect the heterogeneity underlying myeloid cells and, if possible, should be combined with mass cytometry to profile cell-surface markers that characterize MDSCs. ${ }^{79}$ To delineate monocyte-tomacrophage differentiation, we performed a trajectory inference analysis. We excluded non-classical monocytes and related macrophages $(\mathrm{C} 2, \mathrm{C} 7)$, and resident macrophages (C8, C9). In the trajectory, C1 monocytes were progenitor cells for C3 immature macrophages (Fig. 7f). Next on the time scale were C4 macrophages, which further separated into C5 and C6 macrophages, suggesting C4 macrophages to be endowed with high plasticity prior to M2 differentiation. Interestingly, most of LC macrophages differentiated into both lineages (Supplementary information, Fig. S7k). Profiling of gene expression dynamics along the trajectory (Fig. $7 \mathrm{~g}, \mathrm{~h}$ ) revealed a reduction of known monocyte markers (CD14, S100A8, SELL) and increased expression of 230 other genes (Supplementary information, Table S9), including several M2 markers. SCENIC identified several TFs underlying each myeloid phenotype or the monocyte-to-macrophage differentiation trajectory (Fig. 7i, j; Supplementary information, S7l, m). For example, there was a gradual increase of MAFB and decrease of FOS, FOSB and EGR1 along the trajectory, as reported. ${ }^{80,81}$ Interestingly, terminally differentiated clusters $(C 5, C 6)$ were characterised by distinct TFs, but also shared TFs, including the hypoxia-induced HIF$2 a^{82}$ (EPAS1; Supplementary information, Fig. S7n).

Finally, we also identified 1962 mast cells. These cells represent a rare stromal cell type that was not enriched for in tumors, and that could be subclustered into 4 cellular phenotypes (Supplementary information, Fig. S8a-h).

\section{Mapping the blueprint in breast cancer}

In 3 different cancers, we identified 68 stromal cell (sub)types, of which 46 were shared. To confirm this heterogeneity in another cancer type, we profiled 14 treatment-naïve breast cancers (BC) using $5^{\prime}$-scRNA-seq and clustered the 44,024 cells with high quality data (Materials and methods). After assigning cell types (Fig. 8a; Supplementary information, Fig. S9a), we re-clustered cells per cell type using unaligned clustering, or after pooling cell type data from $B C$ with those from other cancer types, while applying CCA alignment for $5^{\prime}$ versus $3^{\prime}$-scRNA-seq. Both approaches clustered the 14,413 T-cells from BC into their 10 cellular phenotypes, each with similar expression signatures as described for 3'-scRNA-seq (Fig. 8b; Supplementary information, Fig. S9b). However, in other cell types unaligned clustering failed to identify the cellular phenotypes, especially when they were less abundant. In contrast, CCA recovered 43 out of the 46 shared phenotypes (Fig. 8b, c; Supplementary information, Fig. S9c). Only for mast cells, for which too few cells were detected $(n=360)$, CCA also failed to identify the respective phenotypes. Notably, across cancer types all cellular phenotypes were characterised by a highly similar expression of marker genes and underlying TFs (Fig. 8d, e; Supplementary information, Fig. S9d-h). These data confirm that the stromal cell blueprint can also be assigned to other cancer types.

When subsequently comparing stromal cell type distribution between BC and all other cancers, we found more T-cells in BC than CRC or OvC, but not LC (Supplementary information, Fig. S9i). At the subcluster level, BC was enriched for pDCs (C4_LILRA4), but had few lymphatic ECs (C5_PROX1; Supplementary information, Fig. S9j). This is possibly because most patients (8/14) had a triplenegative $B C$, which is more immunogenic, without lymph node involvement.

The blueprint as a guide to interpret scRNA-seq studies We also applied our blueprint to SMART-seq2 data from melanomas treated with immune checkpoint inhibitors (ICls). We clustered our T-/NK-cells from the blueprint with the 12,681 T-/NKcells profiled by SMART-seq $2,{ }^{8}$ while performing CCA for technology. This resulted in the $10 \mathrm{~T}$-/NK-cell phenotypes of the blueprint (Supplementary information, Fig. S10a-c). Cells profiled by both technologies contributed to every phenotypic T-/NK-cell cluster, each with similar expression signatures, suggesting effective CCA alignment. Next, we confirmed findings of SadeFeldman et al., ${ }^{8}$ showing that (i) presence of exhausted $\mathrm{CD}^{+} \mathrm{T}$ cells (C1) in melanoma tumors predicts resistance to $\mathrm{ICl}$, while (ii) increased expression of the naïve T-cell marker TCF7 across CD8 ${ }^{+}$ T-cells predicts response to $\mathrm{ICl}$ (Supplementary information, Fig. S10d). However, when assessing TCF7 in the context of the blueprint, we found it was expressed in 2 out of $4 \mathrm{CD}^{+}{ }^{+}$T-cell phenotypes (C2-C3), of which only pre-effector CD8 ${ }^{+}$T-cells (C2) were significantly more prevalent in responders (Fig. $8 \mathrm{f}, \mathrm{g}$ ). Additionally, TCF7 expression was high in naïve CD4 ${ }^{+}$T-cells (C5), which were also enriched in responders $(P=0.0021)$. Receiver operating characteristic (ROC) analysis to evaluate the predictive effect of the C5 cluster revealed an AUC of $0.90(P=0.0021$; Fig. 8h). Albeit to a lesser extent, $C 1$ and $C 2$ clusters were also enriched in non-responders and responders, respectively (Supplementary information, Fig. S10e). Notably, CD4 ${ }^{+} \mathrm{TCF}^{+}$T-cells resided outside of blood vessels, within the tumor at the peritumoral front (Supplementary information, Fig. S10f). 
a

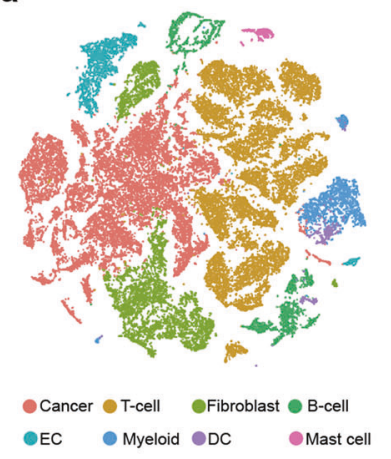

b

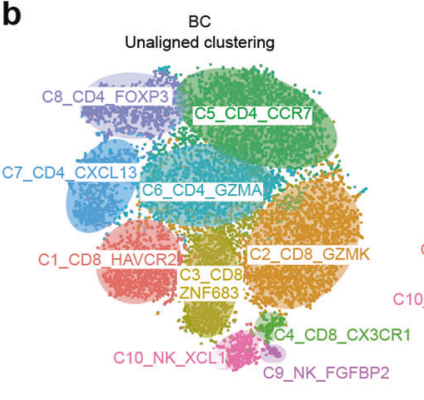

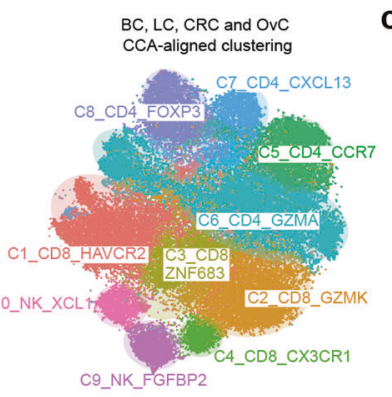

C1_CD8_HAVCR2 C2_CD8_GZMK C3_CD8_ZNF683 C4_CD8_CX3CR1

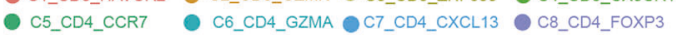
- C9_NK_FGFBP2 C10_NK_XCL1

$\mathbf{e}$

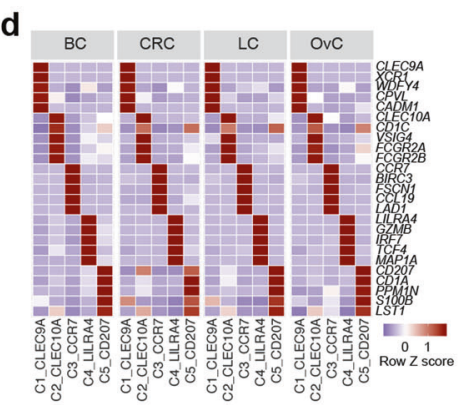

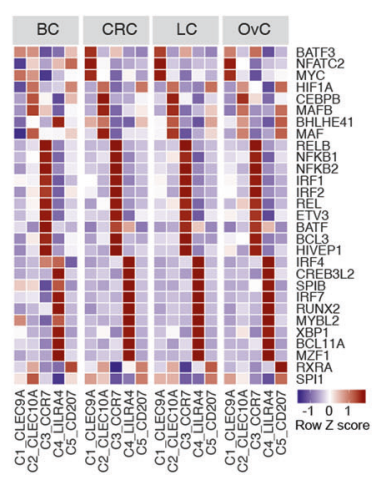

f

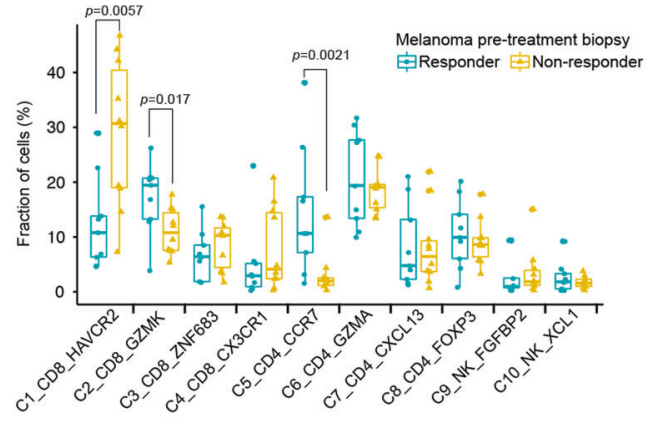

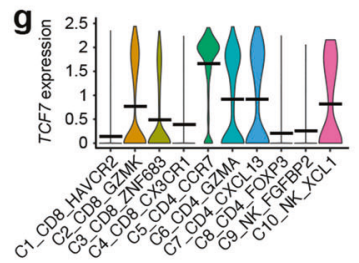

h

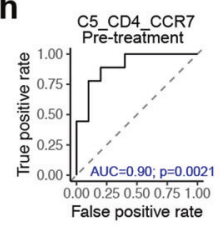

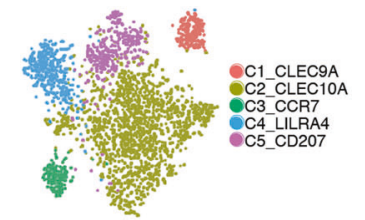

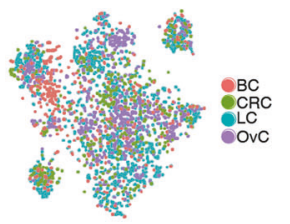

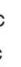

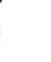

Fig. 8 Validation of the stromal blueprint. a t-SNE of BC cells colour-coded for cell types. $\mathbf{b}$ t-SNEs of T-/NK-cells by unaligned clustering or CCA-aligned clustering with 3'-sCRNA-seq data. c t-SNEs of CCA-aligned clusters colour-coded for annotated DCs (upper) and cancer type (lower). d Heatmap of marker gene expression across DC clusters in different cancer types. e TF activity across DC subclusters in different cancer types. f Fraction of T-/NK-cell clusters in pre-treatment biopsies from melanoma patients treated with ICI. $\mathbf{g}$ Violin plot showing TCF7 expression in T-/NK-cell clusters from pre-treatment melanoma patients. $\mathbf{h}$ ROC analysis to evaluate the predictive effect of naïve CD4 ${ }^{+}$T-cells on response to checkpoint immunotherapy. The area under the ROC curve (AUC) was used to quantify response prediction.

Next, we applied our blueprint to monitor changes in T-/NKcells during $\mathrm{ICl}$. When comparing pre- versus on-treatment biopsies ( $n=4$ with response versus $n=6$ without response), we observed an increase in exhausted $\mathrm{CD}^{+}$T-cells (C1_CD8_HAVCR2) in on-treatment biopsies. Vice versa, there was a relative decrease in naïve $\mathrm{CD}^{+}{ }^{-}$(C5_CD4_CCR7) T-cells (Supplementary information, Fig. S10g, h). Notably, these differences were only observed in responding patients, suggesting that during response, phenotypic clusters that predict resistance in the pre-treatment biopsy increase, while those predicting response decrease in prevalence. Overall, these data illustrate that singlecell data obtained with various technologies can be re-analysed in the context of the blueprint.

Validation of the blueprint at protein level

With the availability of CITE-seq, we can now simultaneously detect RNA and protein expression at single-cell level. ${ }^{83}$ To confirm the cancer blueprint at protein level, a panel of 198 antibodies (Supplementary information, Table S10) compatible with 3'-scRNA-seq was used. We processed 5 BCs, obtaining 6,194 cells with both transcriptome and proteome data. Independent clustering of both datasets revealed how cell types could be discerned based on either marker gene or protein expression (Fig. 9a, b). Since antibodies were mainly directed against immune cells, especially T-cells, we focused our subclustering efforts on this cell type. We pooled 1310 T-/NK-cells with both RNA and protein data together with T-/NK-cells from the blueprint. Subsequent clustering based on scRNA-seq data accurately assigned each T-/NK-cell to its phenotypic cluster (Fig. 9c, d). Next, we selected marker genes amongst the 198 antibodies and explored protein expression per cluster (Fig. 9e). A combination of CD3, CD4, CD8 and NCR1 effectively discriminated CD4 ${ }^{+}, \mathrm{CD}^{+}{ }^{+} \mathrm{T}-$ cells and NK-cells. The T-cell exhaustion marker PD-1 discriminated exhausted $\mathrm{CD}^{+}$and $\mathrm{CD} 8^{+}$T-cell phenotypes $(\mathrm{C} 1, \mathrm{C} 7)$, while IL2RA (CD25) was specific for CD4 ${ }^{+}$Tregs (C8). CD8 ${ }^{+}$memory T-cells (C3) were characterised by high ITGA1 but low PDCD1. Both the cytotoxic T-/NK-cells $(C 4, C 9)$ had high levels of KLRG1, while CD4 ${ }^{+}$ naïve cells had high ITGA6 and SELL (C5). Unfortunately, there were no antibodies specific for C2 and C6 cells. Despite this limitation, a random forest model developed to predict major cell types and T-cell phenotypes based on CITE-seq classified $>80 \%$ of cells into the same cell (sub)type compared to scRNA-seq data.

\section{DISCUSSION}

Here, we performed scRNA-seq on 233,591 single cells from 36 patients with either lung, colon, ovarian or breast cancer. By applying two different clustering approaches -one designed to 

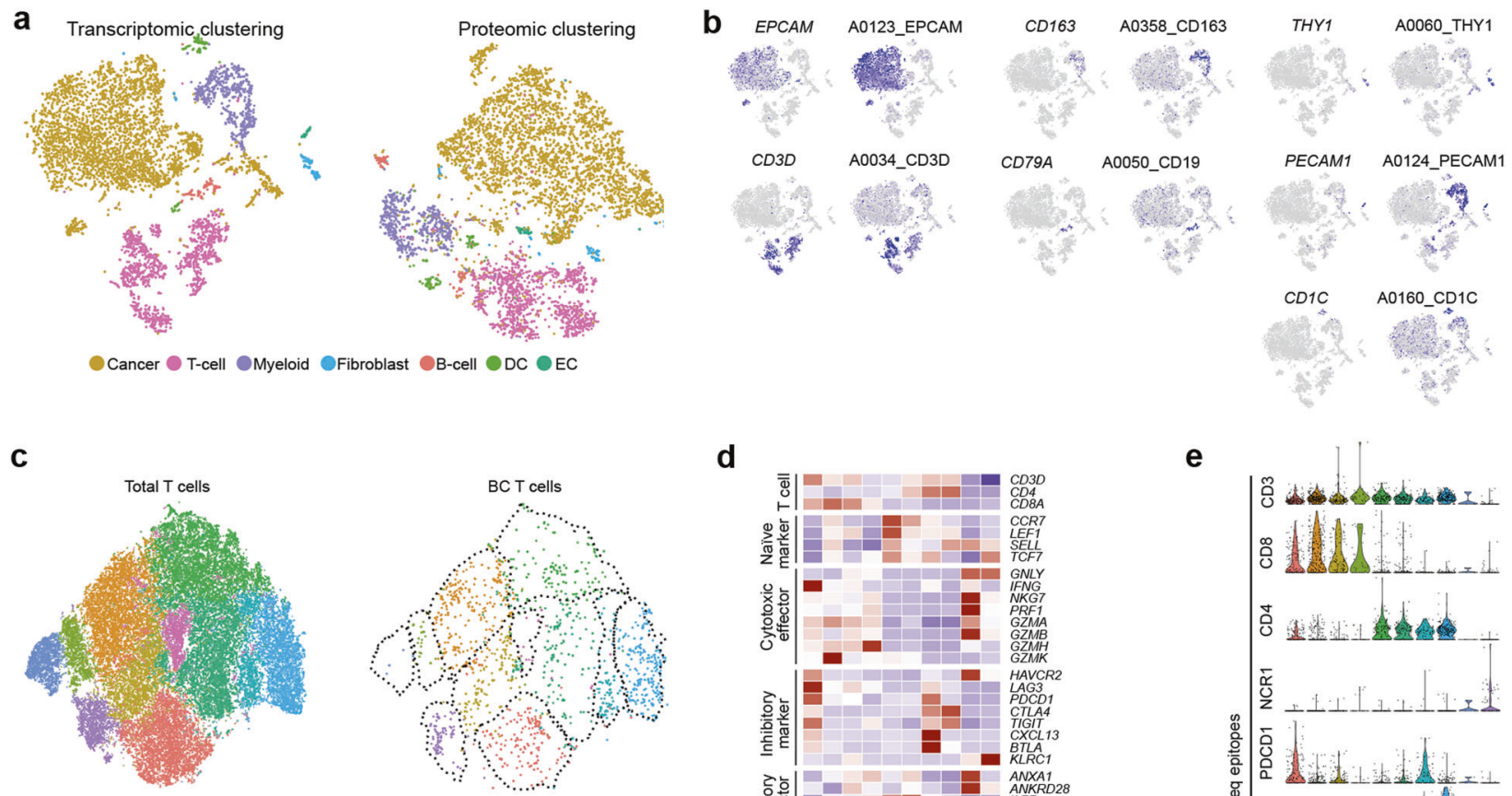

d
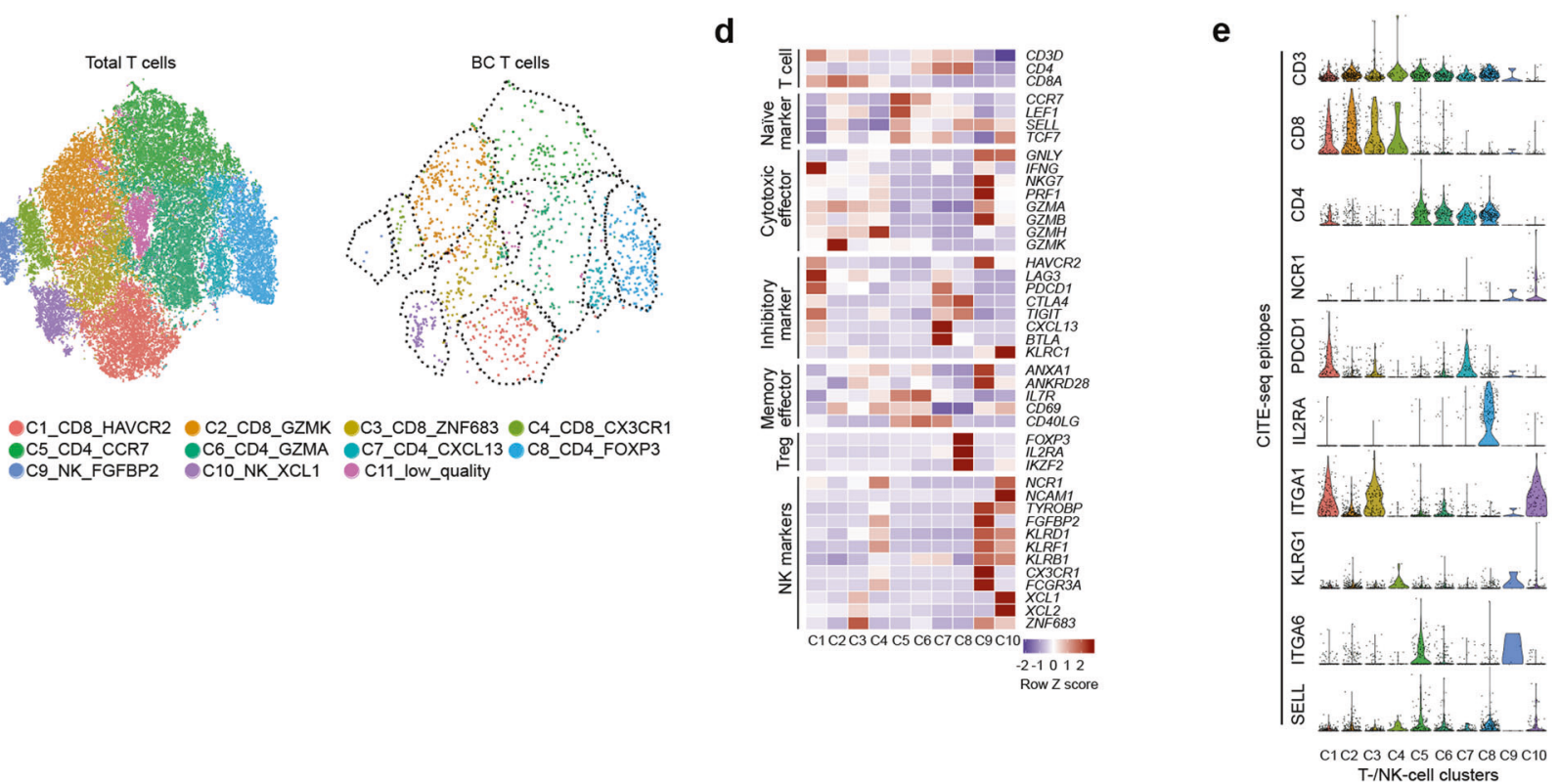

Fig. 9 Validation of the stromal blueprint by CITE-seq. a t-SNEs of CITE-seq profiled BC cells clustered into cell types based on RNA (left) or protein (right) data. b Marker gene or protein expression for each cell type. c t-SNE plots showing BC T-/NK-cells co-clustered with $3^{\prime}$-scRNAseq data from other cancer types (left), while highlighting only T-/NK-cells with BC origin (right). d Heatmap with marker gene expression of T-/NK-cell clusters. e Expression by CITE-seq markers per T-/NK-cell cluster.

detect tissue-specific differences, the other to find shared heterogeneity amongst stromal cell types- we constructed a pan-cancer blueprint of stromal cell heterogeneity. Briefly, we found that tissue-resident cell types, including ECs and fibroblasts, were characterised by considerable patient and tissue specificity in the normal tissue, but that part of this heterogeneity disappeared within the TME. On the other hand, phenotypes involving non-residential cell types, which encompass most of the tumor-infiltrating immune cells, were often shared amongst all patients and cancer types. Overall, we identified 68 stromal phenotypes, of which 46 were shared between cancer types and 22 were cancer type-unique. Amongst the shared phenotypes, several have not previously been described at single-cell level, including tumor-associated pericytes and other fibroblast phenotypes, mast cells, GC-independent B-cells, neutrophils, etc. Of note, by applying a CITE-seq approach to simultaneously profile gene and protein expression, we confirmed all major cell types and Tcell phenotypes identified by scRNA-seq.

An important merit of our study is the public availability of the scRNA-seq data and the stromal blueprint we describe, which can all be interactively accessed via our blueprint server. This will allow scientists to co-cluster their own scRNA-seq data together with blueprint data and assign each of their individual cells to a cellular phenotype. This can also be achieved by feeding our stromal blueprint dataset to established machine learning pipelines, e.g., CellAssign, ${ }^{84}$ and assigning each new cell to the most likely proxy.
Such strategy would indeed be highly relevant, as several of our cellular phenotypes are missed when a smaller number of cells is analysed. Interestingly, as illustrated for melanoma, pooling new with existing scRNA-seq data was even possible when a different single-cell technology was used. Similarly, this blueprint could serve as training matrix to estimate the prevalence from specific cell (sub)types in bulk tissue transcriptomes using newly developed deconvolution methods, i.e., CIBERSORTx. ${ }^{85}$ This is important, as bulk RNA-seq data of tumor tissues are often available for multiple large and homogeneous cohorts of cancer patients.

We also built trajectories between relevant cell phenotypes, highlighting how several of these do not represent separate entities. Stratification of these trajectories for cancer type revealed some intriguing differences. For instance, LC contained more exhausted $\mathrm{CD}^{+}$cytotoxic T-cells in the C1_CD8_HAVCR2 trajectory. Moreover, LC appeared more inflammatory as it was enriched for differentiated myeloid cells along both the CCL18 and MMP9 lineage. Also, memory B-cells were more differentiated in LC, while CDC2s got stuck early in the trajectory in OvC. Most probably, these differences are due to the fact that LC is an immune-infiltrated cancer with a high tumor mutation burden (TMB) and neoepitope load, ${ }^{86}$ while OvC and CRC are cold tumors with a low TMB.

We believe our blueprint is also useful when monitoring dynamic changes in the TME during cancer treatment. Indeed, by 
performing scRNA-seq on individual biopsies obtained before and during treatment, individual cells can be assigned to each phenotypic cluster and changes can easily be interpreted in the context of the blueprint. For instance, when re-analysing a set of pre- versus on-treatment biopsies from melanomas exposed ICls, we observed that exhausted $\mathrm{CD} 8^{+}$T-cells became gradually more common during treatment, while naïve $\mathrm{CD} 4^{+} \mathrm{T}$-cells became less common. Notably, these shifts were only observed in patients responding to the treatment. Although findings that naïve $\mathrm{CD}_{4}^{+}$ helper T-cells predict checkpoint immunotherapy are novel, these findings are not unexpected. Firstly, $\mathrm{CD}^{+}$helper T-cells can also express PD1, and are thus targeted by the treatment. Furthermore, they can enhance $\mathrm{CD}^{+}$T-cell infiltration, ${ }^{87}$ improve antibody penetration, ${ }^{88}$ T-cell memory formation, or have a direct cytolytic capacity. ${ }^{89}$ Several other studies suggest the role of both naïve $\mathrm{CD}^{+}$and $\mathrm{CD}^{+}$T-cells in priming anti-tumor activity. ${ }^{90}$ Overall, we believe that our approach to monitor how blueprint phenotypes change in response to cancer treatment and gradually also contribute to therapeutic resistance, will allow scientists to gain important insights into the mechanisms of action of novel cancer drugs.

\section{MATERIALS AND METHODS}

Patients

This study was approved by the local ethics committee at the University Hospital Leuven for each cancer type. Only patients provided with informed consent were included in this study. The clinical information of all patients was summarised in Supplementary information, Table S1.

\section{Preparation of single-cell suspensions}

Following resection, samples from the tumor and adjacent nonmalignant tissue were rapidly processed for single-cell RNAsequencing. Samples were rinsed with $\mathrm{PBS}$, minced on ice to pieces of $<1 \mathrm{~mm}^{3}$ and transferred to $10 \mathrm{~mL}$ digestion medium containing collagenase $\mathrm{P}\left(2 \mathrm{mg} \mathrm{mL}^{-1}\right.$, ThermoFisher Scientific) and DNAse I (10U $\mu \mathrm{L}^{-1}$ Sigma) in DMEM (ThermoFisher Scientific). Samples were incubated for $15 \mathrm{~min}$ at $37^{\circ} \mathrm{C}$, with manual shaking every $5 \mathrm{~min}$. Samples were then vortexed for $10 \mathrm{~s}$ and pipetted up and down for 1 min using pipettes of descending sizes $(25,10$ and $5 \mathrm{~mL}$ ). Next, $30 \mathrm{~mL}$ ice-cold PBS containing $2 \%$ fetal bovine serum was added and samples were filtered using a $40 \mu \mathrm{m}$ nylon mesh (ThermoFisher Scientific). Following centrifugation at $120 \times g$ and $4{ }^{\circ} \mathrm{C}$ for $5 \mathrm{~min}$, the supernatant was decanted and discarded, and the cell pellet was resuspended in red blood cell lysis buffer. Following a 5-min incubation at room temperature, samples were centrifuged $\left(120 \times \mathrm{g}, 4^{\circ} \mathrm{C}, 5 \mathrm{~min}\right)$ and resuspended in $1 \mathrm{~mL}$ PBS containing $8 \mu \mathrm{L}$ UltraPure BSA $\left(50 \mathrm{mg} / \mathrm{mL}^{-1}\right.$; AM2616, ThermoFisher Scientific) and filtered over Flowmi $40 \mu \mathrm{m}$ cell strainers (VWR) using wide-bore $1 \mathrm{~mL}$ low-retention filter tips (MettlerToledo). Next, $10 \mu \mathrm{L}$ of this cell suspension was counted using an automated cell counter (Luna) to determine the concentration of live cells. The entire procedure was completed in less than $1 \mathrm{~h}$ (typically about $45 \mathrm{~min}$ ).

\section{Single cell RNA-seq data acquisition and pre-processing}

Libraries for scRNA-seq were generated using the Chromium Single Cell $3^{\prime}$ or $5^{\prime}$ library and Gel Bead \& Multiplex Kit from 10x Genomics (Supplementary information, Table S2). We aimed to profile 5000 cells per library (if sufficient cells were retained during dissociation). All libraries were sequenced on Illumina NextSeq HiSeq4000 or NovaSeq6000 until sufficient saturation was reached (73.8\% on average, Supplementary information, Table S2). After quality control, raw sequencing reads were aligned to the human reference genome GRCh38 and processed to a matrix representing the UMI's per cell barcode per gene using CellRanger (10x Genomics, v2.0).
Single-cell RNA analysis to determine major cell types and cell phenotypes

Raw gene expression matrices generated per sample were merged and analysed with the Seurat package (v2.3.4). Matrices were filtered by removing cell barcodes with $<401$ UMls, $<201$ expressed genes, $>6000$ expressed genes or $>25 \%$ of reads mapping to mitochondrial RNA. The remaining cells were normalized and genes with a normalized expression between 0.125 and 3 , and a quantile-normalized variance $>0.5$ were selected as variable genes. The number of variably-expressed genes differs for each clustering step (Supplementary information, Table S4). When clustering cell types, we regressed out confounding factors: number of UMIs, percentage of mitochondrial RNA, patient ID and cell cycle (S and G2M phase scores calculated by the CellCycleScoring function in Seurat). After regression for confounding factors, all variably-expressed genes were used to construct principal components (PCs) and PCs covering the highest variance in the dataset were selected. The selection of these PCs was based on elbow and Jackstraw plots. Clusters were calculated by the FindClusters function with a resolution between 0.2 and 2 , and visualised using the t-SNE dimensional reduction method. Differential gene-expression analysis was performed for clusters generated at various resolutions by both the Wilcoxon rank sum test and Model-based Analysis of Single-cell Transcriptomics (MAST) using the FindMarkers function. A specific resolution was selected when known cell types were identified as a cluster at a given resolution, but not at a lower resolution (Supplementary information, Table S5), with the minimal constraint that each cluster has at least 10 significantly differentially expressed genes (FDR $<0.01$ with both methods) with at least a 2-fold difference in expression compared to all other clusters. Annotation of the resulting clusters to cell types was based on the expression of marker genes (Supplementary information, Fig. S1c). All major cell types were identified in one clustering step, except for DCs; pDCs co-clustered with B-cells, while other DCs co-clustered with myeloid cells. Therefore, we first separated DCs per cancer type based on established marker genes (pDC: LILRA4 and CXCR3; CCDs: CLEC9A, XCR1, CD1C, CCR7, CCL17, CCL19, Langerhans-like: $C D 1 A, C D 207)^{2,40}$ and then pooled these DCs for subclustering.

Next, all cells assigned to a given cell type per cancer type were merged and further subclustered into functional phenotypes using the same strategy, which we refer to as the unaligned clustering approach in the manuscript. However, the confounding factors used for cell types were not sufficient to reduce patientspecific effects when performing the subclustering. Instead of directly applying an unsupervised batch correction algorithm, we found that the interferon response (BROWNE_INTERFERON_RESPONSIVE GENES in the Molecular Signatures Database or MSigDB v6.2) and the sample dissociation-induced gene signatures $^{91}$ represent common patient-specific confounders, which were therefore regressed out. We additionally regressed out the hypoxia signature ${ }^{92}$ for myeloid cells to avoid clusters driven by hypoxia state instead of its origin or (anti-)inflammatory functions. Since hemoglobin and immunoglobulin genes are common contaminants from ambient RNA, hemoglobin genes were excluded for PCA. This also applied to immunoglobulin genes, except when subclustering B-cells. For T-cell subclustering, variable genes of T-cell receptor (TRAVs, TRBVs, TRDVs, TRGVs) were excluded to avoid somatic hypermutation associated variances. Similarly, variable genes of B-cell receptor (IGLVs, IGKVs, IGHVs) were all excluded when subclustering B-cells.

To reveal similarities between the subclusters across cancer types, we performed canonical correlation analysis (CCA, RunMultiCCA function) by aligning data from different cancer types into a subspace with the maximal correlation. ${ }^{11}$ The selection of CCA dimensions or canonical correction vectors (CCs) for subspace alignment were guided by the CC bicor saturation plot 
(MetageneBicorPlot function). Resolution was determined similar to the PCA-based approach described above, followed by marker gene-based cluster annotation. Since CCA is designed to identify shared clusters, we performed CCA alignment without cancer-type specific cells defined by PCA-based approach for fibroblasts and myeloid cells. Low quality clusters were identified based on the number of detected genes within subclusters and the lack of marker genes. Doublet clusters expressed marker genes from other cell lineages, and had a higher than expected (3.9\% according to the User Guide from 10x Genomics) doublets rate, as predicted by the artificial knearest neighbours algorithm implemented in DoubletFinder (v1.0)..$^{93}$ We also used Scrublet ${ }^{94}$ to identify doublet cells and could predict the same clusters as predicted by DoubletFinder. As an example, we evaluate for each of the B-cell clusters, (i) the expression of marker genes from other cell types, (ii) the higher number of detected genes, and (iii) the overlap of cells predicted to be doublets by DoubletFinder and Scrublet (Supplementary Information, Fig. S11a-d).

For a comprehensive statistical analysis, we used a single-cell specific method based on mixed-effects modelling of associations of single cells (MASC) ${ }^{95}$. The analysis systematically addressed two major questions: which cell types are enriched or depleted in all cancers or in a particular cancer type, and which cell types or stromal phenotypes are enriched or depleted in tumors versus normal tissues in all cancers or in a particular cancer type. Events with FDR $<0.05$ were considered significant as summarised in Supplementary information, Table S6.

\section{SCENIC analysis}

Transcription factor (TF) activity was analysed using SCENIC (v1.0.0.3) per cell type with raw count matrices as input. The regulons and TF activity (AUC) for each cell were calculated with the pySCENIC (v0.8.9) pipeline with motif collection version mc9nr. The differentially activated TFs of each subcluster were identified by the Wilcoxon rank sum test against all the other cells of the same cell type. TFs with log-fold-change $>0.1$ and an adjusted $P<1 \mathrm{e}-5$ were considered as significantly upregulated.

Trajectory inference analysis

We applied the Monocle (v2.8.0) algorithm to determine the potential lineage between diverse stromal cell phenotypes. ${ }^{96}$ Seurat objects were imported to Monocle using importCDS function. DDRTree-based dimension reduction was performed with conserved and differentially expressed genes. These genes were calculated for each subcluster across LC, CRC and OvC using FindConservedMarkers function in Seurat using the metap ( $v 1.0$ ) algorithm and Wilcoxon rank sum test (max_pval $<0.01$, minimum_p_val $<1 \mathrm{e}-5)$. PC selection was determined using the PC variance plot (plot_pc_variance_explained function in Monocle, 3-5 PCs). Genes with branch-dependent expression dynamics were calculated using the BEAM test in Monocle. Genes with a $q<$ $1 \mathrm{e}-10$ were plotted in heatmaps. The dynamics of transcription factor activity (or AUC) was calculated by SCENIC and plotted per branch of trajectory along the pseudotime calculated by Monocle. For each TF, the AUC and pseudotime, smoothed as a natural spline using sm.ns function, were fitted in vector generalised linear model (VGLM) using VGAM package v1.1. TF with $q<1 \mathrm{e}-50$ were selected for plotting. Two other trajectory inference pipelines, i.e., Slingshot and SCORPIUS, ${ }^{97,98}$ were also used. Since SCORPIUS cannot handle branched trajectories, we analysed both trajectories separately with the branching topology informed by Monocle analysis. To assess consistency between these pipelines, scaled pseudotime between Monocle, Slingshot and SCORPIUS were compared and high correlations were consistently observed between all lineages. Additionally, we compared expression of key marker genes along the trajectories of all 3 tools (Supplementary Information, Fig. S12a-k).
Metabolic and cancer hallmark pathways and geneset enrichment analysis

Metabolic pathway activities were estimated with gene signatures from a curated database. ${ }^{99}$ For robustness of the analysis, lowly expressed genes ( $<1 \%$ cells) or genes shared by multiple pathways were trimmed. And pathways with less than 3 genes were excluded. Cancer hallmark gene sets from Molecular Signatures Database (MSigDB v6.1) were used. The activity of individual cells for each gene set was estimated by AUCell package (v1.2.4). The differentially activated pathways of each subcluster were identified by running the Wilcoxon rank sum test against other cells of the same cell type. Pathways with log-foldchange $>0.05$ and an adjusted $P<0.01$ were considered as significantly upregulated. GO and REACOTOME geneset enrichment analyses were performed using hypeR package, ${ }^{100}$ geneset over-representation was determined by hypergeometric test.

\section{CITE-seq}

We adopted the established CITE-seq protocol $^{83}$ with some modifications. Briefly, 100,000-500,000 single cells of breast tumors were suspended in $100 \mu \mathrm{L}$ staining buffer $(2 \% \mathrm{BSA}$, $0.01 \%$ Tween in PBS) before adding $10 \mu \mathrm{L}$ Fc-blocking reagent (FCX, BioLegend). and incubating during $10 \mathrm{~min}$ on ice. This was followed by the addition of $25 \mu \mathrm{L}$ TotalSeq-A (Biolegend) antibody-oligo pool (1:1000 diluted in staining buffer) and another 30 min incubation on ice. Cells were washed 3 times with staining buffer and filtered through a $40 \mu \mathrm{m}$ flowmi strainer before processing with $3^{\prime}$-scRNA-seq library kits. ADT (Antibody-Derived Tags) additive primers were added to increase the yield of the ADT product. ADT-derived and mRNA-derived CDNAs were separated by SPRI purification and amplified for library construction and subsequent sequencing. For each cell barcode detected in the corresponding RNA library, ADTs were counted in the raw sequencing reads of CITE-seq experiments using CITE-seq-Count version 1.4. In the resulting UMI per ADT matrix, the noise level was calculated for each cell by taking the average signal increased with $3 \times$ the standard deviation of 10 control probes. Signals below this level were excluded. We divided the UMIs by the total UMI count for each cell to account for differences in library size and a centred log-ratio (CLR) normalization specific for each gene was computed. Clustering of protein data was performed using the Euclidean distance matrix between cells and t-SNE coordinates were calculated using this distance matrix. The random forest algorithm incorporated in Seurat was iteratively applied on a training and test set, consisting of $67 \%$ and $33 \%$ of cells respectively, to predict cell types and T-/NK-cell phenotypes.

Immunofluorescence assay and analysis

A 5- $\mu \mathrm{m}$-section of a formalin-fixed, paraffin-embedded (FFPE) microarray containing 14 melanoma metastasis from 9 patients was stained with antibodies against SOX10 (SCBT; sc-365692), CD4 (abcam; ab133616), CD31 (LSBio; LS-C173974) and TCF7 (R\&D systems; AF5596) at a concentration of $1 \mu \mathrm{g} / \mathrm{mL}$ according to the Multiple Iterative Labeling by Antibody Neodeposition (MILAN) protocol, as described. ${ }^{101}$

Tumor mutation detection

Whole-exome sequencing was performed as described previously. ${ }^{102}$ The average sequencing depth was $\times 161 \pm 67$ coverage. Mutation of CRC samples were detected using Illumina Trusight26 Tumour kit.

\section{DATA AVAILABILITY}

Raw sequencing reads of the single-cell RNA experiments have been deposited in the ArrayExpress database at EMBL-EBI (www.ebi.ac.uk/arrayexpress) under accession number E-MTAB-8107, E-MTAB-6149 and E-MTAB-6653. Based on SCope package, ${ }^{103}$ 
an interactive web server for scRNA-seq data visualisation, exploration and downloading of the count matrix is available at http://blueprint.lambrechtslab.org.

\section{ACKNOWLEDGEMENTS}

We thank T. Van Brussel, R. Schepers, and E. Vanderheyden for their technical assistance. This work was supported by VIB TechWatch funding, Scientific Fund for Research-Flanders (FWO) grants to D. Lambrechts (G065615N), a Stichting tegen Kanker (STK) grant to D. Lambrechts (FAF-C/2016/876) and a VIB Grand Challenge grant to $\mathrm{D}$. Lambrechts. The computational resources used in this work were provided by the Flemish Supercomputer Center (VSC), funded by the Hercules Foundation and the Flemish Government, Department of Economy, Science and Innovation (EWI).

\section{AUTHOR CONTRIBUTIONS}

J.Q. and D. Lambrechts. designed and supervised the study and wrote the manuscript; J.Q. and B.B. performed data analysis with significant contributions from P.B. and J.X.; I.V., A.S., S.T. and E.W. coordinated sample collection and clinical annotation with assistance from S.O., H.V., E.E., V.P., S.V., A.B., M.V.B., A.F. and G.F.; F.M.B., Y.V.H. and A.A. performed MILAN for melanoma samples. D. Laoui and B.T. contributed with critical data interpretation. All the authors have read the manuscript and provided useful comments.

\section{ADDITIONAL INFORMATION}

Supplementary information accompanies this paper at https://doi.org/10.1038/ s41422-020-0355-0.

Competing interests: The authors declare no competing interests.

\section{REFERENCES}

1. Tirosh, I. et al. Dissecting the multicellular ecosystem of metastatic melanoma by single-cell RNA-seq. Science 352, 189-96 (2016).

2. Lambrechts, D. et al. Phenotype molding of stromal cells in the lung tumor microenvironment. Nat. Med. 24, 1277-1289 (2018).

3. Puram, S. V. et al. Single-cell transcriptomic analysis of primary and metastatic tumor ecosystems in head and neck cancer. Cell 171, 1611-1624 (2017).

4. Aizarani, N. et al. A human liver cell atlas reveals heterogeneity and epithelial progenitors. Nature 572, 199-204 (2019).

5. Venteicher, A. S. et al. Decoupling genetics, lineages, and microenvironment in IDH-mutant gliomas by single-cell RNA-seq. Science 355, eaai8478 (2017).

6. Hovestadt, V. et al. Resolving medulloblastoma cellular architecture by singlecell genomics. Nature 572, 74-79 (2019).

7. Peng, J. et al. Single-cell RNA-seq highlights intra-tumoral heterogeneity and malignant progression in pancreatic ductal adenocarcinoma. Cell Res. 29, 725-738 (2019).

8. Sade-Feldman, M. et al. Defining $T$ cell states associated with response to checkpoint immunotherapy in melanoma. Cell 175, 998-1013 (2018).

9. Parikh, K. et al. Colonic epithelial cell diversity in health and inflammatory bowel disease. Nature 567, 49-55 (2019).

10. Cohen, $M$. et al. Lung single-cell signaling interaction map reveals basophil role in macrophage imprinting. Cell 175, 1031-1044 (2018).

11. Butler, A., Hoffman, P., Smibert, P., Papalexi, E. \& Satija, R. Integrating single-cell transcriptomic data across different conditions, technologies, and species. Nat. Biotechnol. 36, 411-420 (2018).

12. Pusztaszeri, M. P., Seelentag, W. \& Bosman, F. T. Immunohistochemical expression of endothelial markers CD31, CD34, von Willebrand factor, and Fli-1 in normal human tissues. J. Histochem. Cytochem. 54, 385-395 (2006).

13. Müller, A. M., Skrzynski, C., Skipka, G. \& Müller, K.-M. Expression of von Willebrand factor by human pulmonary endothelial cells in vivo. Respiration 69, 526-533 (2002)

14. Dhaun, N. \& Webb, D. J. Endothelins in cardiovascular biology and therapeutics. Nat. Rev. Cardiol. 2019 6, 1 (2019).

15. Strickland, L. A. et al. Plasmalemmal vesicle-associated protein (PLVAP) is expressed by tumour endothelium and is upregulated by vascular endothelial growth factor-A (VEGF). J. Pathol. 206, 466-475 (2005).

16. Rupp, C. et al. IGFBP7, a novel tumor stroma marker, with growth-promoting effects in colon cancer through a paracrine tumor-stroma interaction. Oncogene 34, 815-825 (2015).

17. van Beijnum, J. R. Gene expression of tumor angiogenesis dissected: specific targeting of colon cancer angiogenic vasculature. Blood 108, 2339-2348 (2006).

18. Aibar, S. et al. SCENIC: Single-cell regulatory network inference and clustering. Nat. Methods 14, 1083-1086 (2017).
19. Eelen, G. et al. Endothelial cell metabolism. Physiol. Rev. 98, 3-58 (2018).

20. Kalluri, R. The biology and function of fibroblasts in cancer. Nat. Rev. Cancer 16 582-598 (2016).

21. Kurahashi, M. et al. A functional role for the 'fibroblast-like cells' in gastrointestinal smooth muscles. J. Physiol. 589, 697-710 (2011).

22. Lee, H., Koh, B. H., Peri, L. E., Sanders, K. M. \& Koh, S. D. Purinergic inhibitory regulation of murine detrusor muscles mediated by PDGFRa + interstitial cells. J. Physiol. 592, 1283-1293 (2014).

23. Puddifoot, C. A., Wu, M., Sung, R.-J. \& Joiner, W. J. Ly6h regulates trafficking of Alpha7 nicotinic acetylcholine receptors and nicotine-induced potentiation of glutamatergic signaling. J. Neurosci. 35, 3420-3430 (2015).

24. Kinchen, J. et al. Structural remodeling of the human colonic mesenchyme in inflammatory bowel disease. Cell 175, 372-386 (2018).

25. Fujisawa, M. et al. Ovarian stromal cells as a source of cancer-associated fibroblasts in human epithelial ovarian cancer: a histopathological study. PLoS One 13, 1-15 (2018)

26. Jabara, S. et al. Stromal cells of the human postmenopausal ovary display a distinctive biochemical and molecular phenotype. J. Clin. Endocrinol. Metab. 88, 484-492 (2003).

27. Pisarska, M. D., Barlow, G. \& Kuo, F. T. Minireview: roles of the forkhead transcription factor FOXL2 in granulosa cell biology and pathology. Endocrinology 152, 1199-1208 (2011).

28. Rynne-Vidal, A. et al. Mesothelial-to-mesenchymal transition as a possible therapeutic target in peritoneal metastasis of ovarian cancer. J. Pathol. 242, 140-151 (2017).

29. Saunders, W. B. et al. Coregulation of vascular tube stabilization by endothelial cell TIMP-2 and pericyte TIMP-3. J. Cell Biol. 175, 179-191 (2006).

30. Salzer, M. C. et al. Identity noise and adipogenic traits characterize dermal fibroblast aging. Cell 175, 1575-1590 (2018).

31. Haudenschild, D. R. et al. Enhanced activity of transforming growth factor $\beta 1$ (TGF- $\beta 1$ ) bound to cartilage oligomeric matrix protein. J. Biol. Chem. 286 43250-43258 (2011)

32. Staudacher, J. J. et al. Activin signaling is an essential component of the TGF- $\beta$ induced pro-metastatic phenotype in colorectal cancer. Sci. Rep. 7, 1-9 (2017).

33. Simone, T. \& Higgins, P. Inhibition of SERPINE1 function attenuates wound closure in response to tissue injury: a role for PAl-1 in re-epithelialization and granulation tissue formation. J. Dev. Biol. 3, 11-24 (2015).

34. Ghahary, A. et al. Mannose-6-phosphate/IGF-II receptors mediate the effects of IGF1 -induced latent transforming growth factor $\beta 1$ on expression of type I collagen and collagenase in dermal fibroblasts. Growth Factors 17, 167-176 (2000).

35. Brett, A., Pandey, S. \& Fraizer, G. The Wilms' tumor gene (WT1) regulates Ecadherin expression and migration of prostate cancer cells. Mol. Cancer 12, 1-13 (2013).

36. Volksdorf, T. et al. Tight junction proteins Claudin-1 and Occludin are important for cutaneous wound healing. Am. J. Pathol. 187, 1301-1312 (2017).

37. Chim, S. M. et al. EGFL6 promotes endothelial cell migration and angiogenesis through the activation of extracellular signal-regulated kinase. J. Biol. Chem. 286, 22035-22046 (2011)

38. Orimo, A. et al. Stromal fibroblasts present in invasive human breast carcinomas promote tumor growth and angiogenesis through elevated SDF-1/CXCL12 Secretion. Cell 121, 335-348 (2005).

39. Nabet, B. Y. et al. Exosome RNA unshielding couples stromal activation to pattern recognition receptor signaling in cancer. Cell 170, 352-366 (2017)

40. Villani, A.-C. et al. Single-cell RNA-seq reveals new types of human blood dendritic cells, monocytes, and progenitors. Science 356, eaah4573 (2017).

41. Guilliams, M. et al. Unsupervised high-dimensional analysis aligns dendritic cells across tissues and species. Immunity 45, 669-684 (2016).

42. Merad, M., Ginhoux, F. \& Collin, M. Origin, homeostasis and function of Langerhans cells and other langerin-expressing dendritic cells. Nat. Rev. Immunol. 8 , 935-947 (2008).

43. Chopin, M. et al. Langerhans cells are generated by two distinct PU.1-dependent transcriptional networks. J. Exp. Med. 210, 2967-2980 (2013).

44. Geissmann, F. et al. Retinoids regulate survival and antigen presentation by immature dendritic cells. J. Exp. Med. 198, 623-634 (2003).

45. Wu, C. H., Huang, T. C. \& Lin, B. F. Folate deficiency affects dendritic cell function and subsequent T helper cell differentiation. J. Nutr. Biochem. 41, 65-72 (2017).

46. Salaun, B. et al. Cloning and characterization of the mouse homologue of the human dendritic cell maturation marker CD208/DC-LAMP. Eur. J. Immunol. 33, 2619-2629 (2003).

47. Gatto, D., Wood, K. \& Brink, R. EBI2 operates independently of but in cooperation with CXCR5 and CCR7 to direct B cell migration and organization in follicles and the germinal center. J. Immunol. 187, 4621-4628 (2011)

48. Takemori, T., Kaji, T., Takahashi, Y., Shimoda, M. \& Rajewsky, K. Generation of memory B cells inside and outside germinal centers. Eur. J. Immunol. 44, 1258-1264 (2014). 
49. Shi, G.-X., Harrison, K., Wilson, G. L., Moratz, C. \& Kehrl, J. H. RGS13 regulates germinal center b lymphocytes responsiveness to CXC chemokine ligand (CXCL) 12 and CXCL13. J. Immunol. 169, 2507-2515 (2002).

50. Cyster, J. G. \& Allen, C. D. C. B cell responses: cell interaction dynamics and decisions. Cell 177, 524-540 (2019).

51. Turqueti-Neves, A. et al. B-cell-intrinsic STAT6 signaling controls germinal center formation. Eur. J. Immunol. 44, 2130-2138 (2014).

52. Gustafson, C. E. et al. Limited expression of APRIL and its receptors prior to intestinal IgA plasma cell development during human infancy. Mucosal Immunol. 7, 467-477 (2014).

53. Guo, X. et al. Global characterization of T cells in non-small-cell lung cancer by single-cell sequencing. Nat. Med. 24, 978-985 (2018).

54. Böttcher, J. P. et al. NK cells stimulate recruitment of $\mathrm{CDC1}$ into the tumor microenvironment promoting cancer immune control. Cell 172, 1022-1037 (2018).

55. Savas, P. et al. Single-cell profiling of breast cancer T cells reveals a tissueresident memory subset associated with improved prognosis. Nat. Med. 24, 986-993 (2018)

56. Zheng, C. et al. Landscape of infiltrating t cells in liver cancer revealed by singlecell sequencing. Cell 169, 1342-1356 (2017).

57. Crinier, A. et al. High-dimensional single-cell analysis identifies organ-specific signatures and conserved NK cell subsets in humans and mice. Immunity 49, 971-986 (2018)

58. André, $\mathrm{P}$. et al. Anti-NKG2A mAb is a checkpoint inhibitor that promotes antitumor immunity by unleashing both T and NK cells. Cell 175, 1731-1743 (2018).

59. van Montfoort, $\mathrm{N}$. et al. NKG2A blockade potentiates cd8 t cell immunity induced by cancer vaccines. Cell 175, 1744-1755 (2018).

60. Terawaki, S. et al. IFN-a directly promotes programmed cell death-1 transcription and limits the duration of $\mathrm{t}$ cell-mediated immunity. J. Immunol. 186, 2772-2779 (2011).

61. Ancuta, P. et al. Transcriptional profiling reveals developmental relationship and distinct biological functions of $\mathrm{CD} 16+$ and CD16- monocyte subsets. BMC Genomics 10, 403 (2009).

62. Rőszer, T. Understanding the mysterious M2 macrophage through activation markers and effector mechanisms. Mediators Inflamm. 2015, 1-16 (2015).

63. Zagórska, A., Través, P. G., Lew, E. D., Dransfield, I. \& Lemke, G. Diversification of TAM receptor tyrosine kinase function. Nat. Immunol. 15, 920-928 (2014)

64. Hart, K. M., Bak, S. P., Alonso, A. \& Berwin, B. Phenotypic and functional delineation of nurine CX3CR1 + monocyte-derived cells in ovarian cancer. Neoplasia 11, 564-IN10 (2009).

65. Zheng, J. et al. Chemokine receptor CX3CR1 contributes to macrophage survival in tumor metastasis. Mol. Cancer 12, 141 (2013).

66. Schraufstatter, I. U., Zhao, M., Khaldoyanidi, S. K. \& Discipio, R. G. The chemokine CCL18 causes maturation of cultured monocytes to macrophages in the M2 spectrum. Immunology 135, 287-298 (2012).

67. Steen, K. A., Xu, H. \& Bernlohr, D. A. FABP4/aP2 regulates macrophage redox signaling and inflammasome activation via control of UCP2. Mol. Cell. Biol. 37, e00282-16 (2017).

68. Pan, C. et al. Aldehyde dehydrogenase 2 inhibits inflammatory response and regulates atherosclerotic plaque. Oncotarget 7, 35562-35576 (2016).

69. Lim, H. Y. et al. Hyaluronan receptor LYVE-1-expressing macrophages maintain arterial tone through Hyaluronan-mediated regulation of smooth muscle cell collagen. Immunity 49, 326-341 (2018).

70. Xu, H., Chen, M., Reid, D. M. \& Forrester, J. V. LYVE-1-positive macrophages are present in normal murine eyes. Investig. Opthalmol. Vis. Sci. 48, 2162 (2007).

71. Chakarov, S. et al. Two distinct interstitial macrophage populations coexist across tissues in specific subtissular niches. Science 363, eaau0964 (2019).

72. $\mathrm{Wu}, \mathrm{T}$. et al. Regulating innate and adaptive immunity for controlling SIV infection by 25-hydroxycholesterol. Front. Immunol. 9, 2686 (2018).

73. Hogan, L. E., Jones, D. C. \& Allen, R. L. Expression of the innate immune receptor LILRB5 on monocytes is associated with mycobacteria exposure. Sci. Rep. 6, 21780 (2016).

74. Shojaei, F. et al. Bv8 regulates myeloid-cell-dependent tumour angiogenesis. Nature 450, 825-831 (2007).

75. van Galen, P. et al. Single-cell RNA-Seq reveals AML hierarchies relevant to disease progression and immunity. Cell 176, 1265-1281 (2019).
76. Elyada, E. et al. Cross-species single-cell analysis of pancreatic ductal adenocarcinoma reveals antigen-presenting cancer-associated fibroblasts. Cancer Discov. 9, 1102-1123 (2019).

77. Marvel, D. \& Gabrilovich, D. I. Myeloid-derived suppressor cells in the tumor microenvironment: expect the unexpected. J. Clin. Investig. 125, 3356-3364 (2015).

78. Ryckman, C., Vandal, K., Rouleau, P., Talbot, M. \& Tessier, P. A. Proinflammatory activities of S100: proteins S100A8, S100A9, and S100A8/A9 induce neutrophil chemotaxis and adhesion. J. Immunol. 170, 3233-3242 (2003).

79. Bronte, V. et al. Recommendations for myeloid-derived suppressor cell nomenclature and characterization standards. Nat. Commun. 7, 12150 (2016).

80. Liu, H., Shi, B., Huang, C.-C., Eksarko, P. \& Pope, R. M. Transcriptional diversity during monocyte to macrophage differentiation. Immunol. Lett. 117, 70-80 (2008).

81. Kelly, L. M. MafB is an inducer of monocytic differentiation. EMBO J. 19, 1987-1997 (2000).

82. Hickey, M. M. et al. Hypoxia-inducible factor 2 a regulates macrophage function in mouse models of acute and tumor inflammation. J. Clin. Investig. 120, 2699-2714 (2010).

83. Stoeckius, M. et al. Simultaneous epitope and transcriptome measurement in single cells. Nat. Methods 14, 865-868 (2017).

84. Zhang, A. W. et al. Probabilistic cell-type assignment of single-cell RNA-seq for tumor microenvironment profiling. Nat. Methods 16, 1007-1015 (2019).

85. Newman, A. M. et al. Determining cell type abundance and expression from bulk tissues with digital cytometry. Nat. Biotechnol. 37, 773-782 (2019).

86. Samstein, R. M. et al. Tumor mutational load predicts survival after immunotherapy across multiple cancer types. Nat. Genet. 51, 202-206 (2019).

87. Nakanishi, Y., Lu, B., Gerard, C. \& Iwasaki, A. CD8+ T lymphocyte mobilization to virus-infected tissue requires CD4+ T-cell help. Nature 462, 510-513 (2009).

88. lijima, N. \& Iwasaki, A. Access of protective antiviral antibody to neuronal tissues requires CD4 T-cell help. Nature 533, 552-556 (2016).

89. Quezada, S. A. et al. Tumor-reactive CD4+ T cells develop cytotoxic activity and eradicate large established melanoma after transfer into lymphopenic hosts. J. Exp. Med. 207, 637-650 (2010).

90. Borst, J., Ahrends, T., Babała, N., Melief, C. J. M. \& Kastenmüller, W. CD4+ T cell help in cancer immunology and immunotherapy. Nat. Rev. Immunol. 18, 635-647 (2018).

91. Van Den Brink, S. C. et al. Single-cell sequencing reveals dissociation-induced gene expression in tissue subpopulations. Nat. Methods 14, 935-936 (2017).

92. Buffa, F. M., Harris, A. L., West, C. M. \& Miller, C. J. Large meta-analysis of multiple cancers reveals a common, compact and highly prognostic hypoxia metagene. Br. J. Cancer 102, 428-435 (2010).

93. McGinnis, C. S., Murrow, L. M. \& Gartner, Z. J. DoubletFinder: doublet detection in single-cell RNA sequencing data using artificial nearest neighbors. Cell Syst. 8, 329-337 (2019).

94. Wolock, S. L., Lopez, R. \& Klein, A. M. Scrublet: computational identification of cell doublets in single-cell transcriptomic data. Cell Syst. 8, 281-291.e9 (2019).

95. Fonseka, C. Y. et al. Mixed-effects association of single cells identifies an expanded effector CD4+ T cell subset in rheumatoid arthritis. Sci. Transl. Med. 10, eaaq0305 (2018)

96. Qiu, X. et al. Reversed graph embedding resolves complex single-cell trajectories. Nat. Methods 14, 979-982 (2017).

97. Street, K. et al. Slingshot: cell lineage and pseudotime inference for single-cell transcriptomics. BMC Genomics 19, 1-16 (2018).

98. Cannoodt, R. et al. SCORPIUS improves trajectory inference and identifies novel modules in dendritic cell development. bioRxiv https://doi.org/10.1101/079509 (2016).

99. Gaude, E. \& Frezza, C. Tissue-specific and convergent metabolic transformation of cancer correlates with metastatic potential and patient survival. Nat. Commun. 7, 1-9 (2016).

100. Federico, A. \& Monti, S. hypeR: an R package for geneset enrichment workflows. Bioinformatics 36, 1307-1308 (2019).

101. Bosisio, F. M. et al. Functional heterogeneity of lymphocytic patterns in primary melanoma dissected through single-cell multiplexing. Elife 9, 1-21 (2020).

102. Boeckx, B. et al. The genomic landscape of nonsmall cell lung carcinoma in never smokers. Int. J. Cancer 146, 3207-3218 (2019).

103. Davie, K. et al. A single-cell transcriptome atlas of the aging Drosophila brain. Cell 174, 982-998 (2018). 